\title{
HamleDT: \\ Harmonized Multi-Language Dependency Treebank
}

\author{
Daniel Zeman, Ondřej Dušek, David Mareček, Martin Popel, \\ Loganathan Ramasamy, Jan Štěpánek, Zdeněk Žabokrtský and Jan \\ Hajič \\ Charles University in Prague, Faculty of Mathematics and Physics, ÚFAL
}

February 11, 2014

\begin{abstract}
We present HamleDT - a HArmonized Multi-LanguagE Dependency Treebank. HamleDT is a compilation of existing dependency treebanks (or dependency conversions of other treebanks), transformed so that they all conform to the same annotation style. In the present article, we provide a thorough investigation and discussion of a number of phenomena that are comparable across languages, though their annotation in treebanks often differs. We claim that transformation procedures can be designed to automatically identify most such phenomena and convert them to a unified annotation style. This unification is beneficial both to comparative corpus linguistics and to machine learning of syntactic parsing.
\end{abstract}

Keywords: dependency treebank, annotation scheme, harmonization

\section{Introduction}

Growing interest in dependency parsing is accompanied (and inspired) by the availability of new treebanks for various languages. Shared tasks such as CoNLL 2006-2009 (Buchholz and Marsi, 2006; Nivre et al., 2007; Surdeanu et al., 2008; Hajič et al., 2009) have promoted parser evaluation in multilingual settings. However, differences in parsing accuracy in different languages cannot be always attributed to language differences. They are often caused by variation in domains, sizes and annotation styles of the treebanks. The impact of data size can be estimated by learning curve experiments, but normalizing the annotation style is difficult. We present a method to transform the treebanks into a common style, including a software that implements the method. We have studied treebanks of 29 languages and collected a long list of variations. ${ }^{1}$ We propose one common style (called HamleDT v1.5 style) and provide a transformation from original annotations to this style for almost all ${ }^{2}$ the phenomena we identified. In addition to dependency

1 The initial version has been described in Zeman et al. (2012).

2 HamleDT v1.5 does not include the harmonization of verbal groups (see Section 5.4).

() 2014 Kluwer Academic Publishers. Printed in the Netherlands. 
tree structure normalization, we also unify the tagsets of both the part-of-speech/morphological tags and the dependency relation tags.

The motivation for harmonizing the annotation conventions used for different treebanks was already described in literature, e.g., by McDonald et al. (2013). Clearly, a unified representation of language data is supposed to facilitate the development of multilingual technologies. The harmonized set of treebanks should improve the interpretability and comparability of parsing accuracy results, and thus help to drive the development of dependency parsers towards multilingual robustness. For instance, the range of unlabeled attachment scores reached by a typical state-of-the-art supervised dependency parser in different languages spans an interval of around 10 percent points (given training data of a comparable size) and is even bigger for unsupervised parsers, as documented, e.g., by Mareček and Žabokrtský (2012). It is not entirely clear whether and to what extent this variance can be attributed to the peculiarities of the individual languages, or merely to the choice of annotation conventions used for the language. Using HamleDT should make it possible to separate these two sources of variance. Besides supervised and unsupervised multilingual parsing, homogeneity of the data is also essential for experiments on cross-lingual transfer of syntactic structures, be it based on projecting trees (Hwa et al., 2005) or on transferring delexicalized models (McDonald et al., 2011a).

The common style defined in HamleDT v1.5 serves as a reference point: the ability to say "our results are based on HamleDT v1.5 transformations of treebank XY" will facilitate the comparability of future results published in all these subfields.

The purpose of HamleDT is not to find a single choice of annotation conventions that ideally suits all possible tasks concerning syntactic structures, as this is hardly to be expected doable. However, assuming a different annotation convention fits a particular task better, it is much simpler to transform all the treebanks to the desired shape after they have been collected and unified in HamleDT.

Last but not least, we believe that the unified representation of linguistic content may be advantageous for linguists, enabling them to compare languages based on treebank material without the need to study multiple annotation guidelines.

\section{Related Work}

There have been a few attempts recently to address the same problem, namely: 
- Schwartz et al. (2012) define two measures of syntactic learnability and evaluate them using five different parsers on varying annotation styles of six phenomena (coordination, infinitives, noun phrases, noun sequences, prepositional phrases and verb groups). They work only with English; they generate varying annotations during the conversion of the Penn TreeBank WSJ corpus (Marcus et al., 1993) constituency annotation to dependencies.

- Tsarfaty et al. (2011) compare the performance of two parsers on different constituency-to-dependency conversions of the (English) Penn Treebank. They do not see the solution in data transformations; instead, they develop an evaluation technique that is robust with respect to some ${ }^{3}$ annotation styles.

- McDonald et al. (2011b) experiment with cross-language parser training, relying on a rather small universal set of part-of-speech tags. They do not transform syntactic structures, however. They note that different annotation schemes across treebanks are responsible for the fact that some language pairs work better together than others. They use English as the source language and Danish, Dutch, German, Greek, Italian, Portuguese, Spanish, and Swedish as target languages.

- Seginer (2007) discusses possible annotation schemes for coordination structures and relative clauses in relation to his common cover link representation.

- Bosco et al. (2010) compare three different dependency parsers developed and tested with respect to two Italian treebanks.

- Bengoetxea and Gojenola (2009) evaluate three types of transformations on Basque: transformation of subordinate sentences, coordinations and projectivization. An important difference between their approach and ours is that their transformations can change tokenization.

- Nilsson et al. (2006) show that transformations of coordination and verb groups improve parsing of Czech.

\footnotetext{
3 The transformations are not robust to coordination styles.
} 


\section{Data}

We identified over 30 languages for which treebanks exist and are available for research purposes. Most of them can either be acquired free of charge or are included in the Linguistic Data Consortium ${ }^{4}$ membership fee.

Most of the treebanks are natively based on dependencies, but some were originally based on constituents and transformed via a headselection procedure. For instance, Spanish phrase-structure trees were converted to dependencies using the method of Civit et al. (2006).

HamleDT v1.5 currently covers 29 treebanks, with several others to be added soon. Table I lists the treebanks along with their data sizes. In the following, we use ISO 639 language codes in square brackets to refer to the treebanks of these languages, so e.g. [en] refers to the English treebank. A list of all 29 treebanks with references is included in Appendix A.

Many treebanks (especially those used in CoNLL shared tasks) define a train/test data split. This is important for the comparability of experiments with automated parsing and part-of-speech tagging. We preserve the original data division and define test subsets for the remaining treebanks as well. In doing so, we try to keep the test size similar to the majority of CoNLL 2006/2007 test sets, i.e., roughly 5,000 tokens.

Throughout this article, a dependency tree is an abstract structure of nodes and dependencies that capture syntactic relations in a sentence. Nodes correspond to the tokens of the sentence, i.e. to words, numbers, punctuation and other symbols (see Section 5.7 for more on tokenization). Besides the actual word form, the node typically holds additional attributes of the token, such as its lemma and part of speech. Dependencies are directed arcs between nodes. Every node is attached to (depends on) exactly one other node, called its parent. We draw the dependency as an arrow going from the parent to the child. Thus every node has one incoming dependency and any number of outgoing dependencies. There is one exception: an artificial root node that does not correspond to any real token and has only outgoing dependencies. Dependencies have labels that mark the type of the relation.

Most diagrams in this article (Figure 1 and onwards) depict just a snippet of the sentence, i.e. a subtree. Selected tokens (word forms) are shown in a sequence respecting the word order, with dependencies drawn as labeled arrows between two tokens (nodes). The artificial root of the whole sentence is never shown; the root token of the subtree

\footnotetext{
${ }^{4}$ http://www.ldc. upenn.edu/
} 
has one incoming dependency going straight down (from an invisible parent). The relation between the subtree and its invisible parent is labeled X (it does not make sense to show the real relation type without the parent).

\begin{tabular}{|c|c|c|c|c|c|c|c|}
\hline Language & $\begin{array}{l}\text { Prim. } \\
\text { tree } \\
\text { type }\end{array}$ & $\begin{array}{l}\text { Used } \\
\text { data } \\
\text { source }\end{array}$ & Sents. & Tokens & $\begin{array}{l}\text { Train } \\
\text { / test } \\
{[\% \text { sents }]}\end{array}$ & $\begin{array}{r}\text { Avg. } \\
\text { sent. } \\
\text { length }\end{array}$ & $\begin{array}{r}\text { Nonprj. } \\
\text { deps. } \\
{[\%]}\end{array}$ \\
\hline Arabic (ar) & dep & $\mathrm{C} 2007$ & 3,043 & 116,793 & $96 / 4$ & 38.38 & 0.37 \\
\hline Basque (eu) & dep & prim & 11,226 & 151,604 & $90 / 10$ & 13.50 & 1.27 \\
\hline Bengali (bn) & dep & I2010 & 1,129 & 7,252 & $87 / 13$ & 6.42 & 1.08 \\
\hline Bulgarian (bg) & phr & $\mathrm{C} 2006$ & 13,221 & 196,151 & $97 / 3$ & 14.84 & 0.38 \\
\hline Catalan (ca) & phr & C2009 & 14,924 & 443,317 & $88 / 12$ & 29.70 & 0.00 \\
\hline Czech (cs) & dep & $\mathrm{C} 2007$ & 25,650 & 437,020 & $99 / 1$ & 17.04 & 1.91 \\
\hline Danish (da) & dep & $\mathrm{C} 2006$ & 5,512 & 100,238 & $94 / 6$ & 18.19 & 0.99 \\
\hline Dutch (nl) & phr & $\mathrm{C} 2006$ & 13,735 & 200,654 & $97 / 3$ & 14.61 & 5.41 \\
\hline English (en) & phr & C2007 & 18,577 & 446,573 & $99 / 1$ & 24.03 & 0.33 \\
\hline Estonian (et) & phr & prim & 1,315 & 9,491 & $90 / 10$ & 7.22 & 0.07 \\
\hline Finnish (fi) & dep & prim & 4,307 & 58,576 & $90 / 10$ & 13.60 & 0.51 \\
\hline German (de) & phr & C2009 & 38,020 & 680,710 & $95 / 5$ & 17.90 & 2.33 \\
\hline Greek (el) & dep & $\mathrm{C} 2007$ & 2,902 & 70,223 & $93 / 7$ & 24.20 & 1.17 \\
\hline Greek (grc) & dep & prim & 21,160 & 308,882 & $98 / 2$ & 14.60 & 19.58 \\
\hline Hindi (hi) & dep & I2010 & 3,515 & 77,068 & $85 / 15$ & 21.93 & 1.12 \\
\hline Hungarian (hu) & phr & $\mathrm{C} 2007$ & 6,424 & 139,143 & $94 / 6$ & 21.66 & 2.90 \\
\hline Italian (it) & dep & $\mathrm{C} 2007$ & 3,359 & 76,295 & $93 / 7$ & 22.71 & 0.46 \\
\hline Japanese (ja) & dep & $\mathrm{C} 2006$ & 17,753 & 157,172 & $96 / 4$ & 8.85 & 1.10 \\
\hline Latin (la) & dep & prim & 3,473 & 53,143 & $91 / 9$ & 15.30 & 7.61 \\
\hline Persian (fa) & dep & prim & 12,455 & 189,572 & $97 / 3$ & 15.22 & 1.77 \\
\hline Portuguese (pt) & phr & $\mathrm{C} 2006$ & 9,359 & 212,545 & $97 / 3$ & 22.71 & 1.31 \\
\hline Romanian (ro) & dep & prim & 4,042 & 36,150 & $93 / 7$ & 8.94 & 0.00 \\
\hline Russian (ru) & dep & prim & 34,895 & 497,465 & $99 / 1$ & 14.26 & 0.83 \\
\hline Slovene (sl) & dep & C2006 & 1,936 & 35,140 & $79 / 21$ & 18.15 & 1.92 \\
\hline Spanish (es) & phr & C2009 & 15,984 & 477,810 & $90 / 10$ & 29.89 & 0.00 \\
\hline Swedish (sv) & phr & $\mathrm{C} 2006$ & 11,431 & 197,123 & $97 / 3$ & 17.24 & 0.98 \\
\hline Tamil (ta) & dep & prim & 600 & 95,81 & $80 / 20$ & 15.97 & 0.16 \\
\hline Telugu (te) & dep & I2010 & 1,450 & 5,722 & $90 / 10$ & 3.95 & 0.23 \\
\hline Turkish (tr) & dep & $\mathrm{C} 2007$ & 5,935 & 69,695 & $95 / 5$ & 11.74 & 5.33 \\
\hline
\end{tabular}

Table I.: Overview of data resources included in HamleDT v1.5. The average sentence length is the number of tokens divided by the number of sentences. Varying tokenization schemes obviously influence the numbers; see Section 5.7 for details on the individual languages. The $C$ code in the fourth column means "CoNLL shared task", I means "ICON" and prim means primary (non-shared-task) source. The last column gives the percentage of nodes attached non-projectively. 


\section{Harmonization}

Our effort aims at identifying all syntactic constructions that are annotated differently in different treebanks. Once a particular construction is identified, we can typically find all its instances in the treebank using existing syntactic and morphological tags, i.e., with little or no lexical knowledge. Thanks to this fact, we were able to design algorithms to normalize the annotations of many linguistic phenomena to a single style, which we refer to as the HamleDT v1.5 style.

The HamleDT v1.5 style is mostly derived from the annotation style of the Prague Dependency Treebank (PDT, Hajič et al. (2006)). ${ }^{5}$ This is a matter of convenience, to a large extent: This is the scheme with which the authors feel most at home, and many of the included treebanks already use a style similar to PDT. We do not want to claim that the HamleDT v1.5 style is objectively better than other styles. (Please note, however, that in case of coordination, the HamleDT v1.5 style provides a more expressive power than the other options, as described in Section 5.1.)

The normalization procedure involves both structural transformations and changes to dependency relation labels. While we strive to design the structural transformations to be as reversible as possible, we do not attempt to save all information stored in the dependency labels. The original ${ }^{6}$ labels vary widely across treebanks, ranging from very simple, e.g., NMOD "generic noun modifier" in [en], over standard subject, object, etc. relations, to deep-level functions of Pāninian grammar such as karta and karma (k1 and $\mathrm{k} 2)$ in [hi, bn, te]. ${ }^{i}$ It does not seem possible to unify these tagsets without relabeling whole treebanks manually.

${ }^{5}$ So far, there are only two differences between the PDT style (used in [cs]) and the HamleDT v1.5 style: handling of appositions (see Table III) and marking of conjuncts (in HamleDT, the root of a conjunct subtree is marked as conjunct even if it is a preposition or subordinating conjunction; in PDT, only content words are marked as conjuncts). By conjunct, we mean a member of coordination (unlike Quirk et al. (1985)). By content word, we mean autosemantic word, i.e. a word with a full lexical meaning, as contrasted with auxiliary. Note that PDT also has a more abstract layer of annotation (called tectogrammatical), but in this work, we only use the shallow dependencies (called analytical layer in PDT).

${ }^{6}$ Unless we explicitly say otherwise, we mean by "original" the data source indicated in Table I. It may actually differ from the really original treebank. For instance, some of the CoNLL data underwent a conversion procedure to the CoNLL format from other formats, and some information may have been lost in the process.

7 In the Pạninian tradition, karta is the agent, doer of the action, and karma is the "deed" or patient. See Bharati et al. (1994). 
We use a lossy scheme that maps the dependency labels on the moderately-sized tagset of PDT analytical functions ${ }^{8}$ - see Table II.

\begin{tabular}{|c|c|c|c|c|c|c|c|c|c|c|}
\hline Language & Atr & $\mathrm{Adv}$ & Obj & AuxP & $\mathrm{Sb}$ & Pred & Coord & $\mathrm{AuxV}$ & AuxC & rest \\
\hline Arabic (ar) & 36.5 & 6.4 & 9.1 & 14.2 & 6.3 & 3.1 & 4.0 & 0.0 & 2.3 & 18.2 \\
\hline Basque (eu) & 19.6 & 24.0 & 8.7 & 0.0 & 7.2 & 5.7 & 3.4 & 8.3 & 1.0 & 22.2 \\
\hline Bengali (bn) & 18.2 & 22.7 & 17.9 & 0.0 & 16.6 & 16.7 & 4.9 & 0.0 & 0.0 & 3.0 \\
\hline Bulgarian (bg) & 23.3 & 8.8 & 12.8 & 14.6 & 7.7 & 7.3 & 3.1 & 0.8 & 3.3 & 18.4 \\
\hline Catalan (ca) & 22.4 & 16.7 & 5.2 & 9.9 & 7.4 & 8.1 & 2.9 & 9.3 & 1.8 & 16.4 \\
\hline Czech (cs) & 28.5 & 10.4 & 8.1 & 9.9 & 7.1 & 6.0 & 4.1 & 1.2 & 1.7 & 23.1 \\
\hline Danish (da) & 23.8 & 12.2 & 12.1 & 10.7 & 9.8 & 5.3 & 3.4 & 0.0 & 3.4 & 19.3 \\
\hline Dutch (nl) & 14.1 & 24.7 & 6.8 & 10.3 & 8.5 & 7.4 & 2.1 & 5.2 & 3.7 & 17.2 \\
\hline English (en) & 30.0 & 12.0 & 5.7 & 9.8 & 7.9 & 4.3 & 2.2 & 4.0 & 1.8 & 22.2 \\
\hline Estonian (et) & 12.8 & 25.7 & 6.6 & 5.9 & 13.0 & 14.1 & 1.3 & 2.6 & 0.6 & 17.4 \\
\hline Finnish (fi) & 29.7 & 18.2 & 7.8 & 1.5 & 9.4 & 8.3 & 4.1 & 1.6 & 1.2 & 18.2 \\
\hline German (de) & 31.2 & 11.8 & 10.4 & 10.1 & 7.9 & 5.3 & 2.8 & 0.5 & 1.2 & 18.7 \\
\hline Greek (grc) & 15.4 & 13.0 & 14.2 & 3.8 & 7.7 & 8.6 & 6.5 & 0.0 & 1.4 & 29.4 \\
\hline Greek (el) & 39.8 & 9.9 & 7.5 & 8.3 & 7.1 & 4.5 & 3.2 & 4.0 & 1.6 & 14.0 \\
\hline Hindi (hi) & 26.8 & 13.4 & 9.6 & 21.1 & 6.8 & 5.3 & 2.4 & 6.3 & 1.6 & 6.8 \\
\hline Hungarian (hu) & 30.4 & 13.9 & 5.2 & 1.6 & 5.9 & 8.3 & 2.4 & 1.3 & 1.6 & 29.2 \\
\hline Italian (it) & 22.2 & 12.4 & 4.9 & 14.7 & 5.2 & 4.8 & 3.3 & 2.8 & 1.1 & 28.5 \\
\hline Japanese (ja) & 11.5 & 16.6 & 0.6 & 5.8 & 3.4 & 7.3 & 0.3 & 0.0 & 0.0 & 54.6 \\
\hline Latin (la) & 17.9 & 13.7 & 15.9 & 5.3 & 10.6 & 8.8 & 6.6 & 1.1 & 3.1 & 17.2 \\
\hline Persian (fa) & 25.3 & 8.8 & 10.0 & 14.0 & 6.4 & 7.7 & 4.1 & 0.1 & 2.7 & 20.8 \\
\hline Portuguese (pt) & 24.6 & 24.0 & 7.1 & 11.4 & 6.0 & 4.3 & 2.4 & 0.0 & 1.0 & 19.0 \\
\hline Romanian (ro) & 27.7 & 13.3 & 7.2 & 17.6 & 8.5 & 11.2 & 1.8 & 7.7 & 0.0 & 5.0 \\
\hline Russian (ru) & 30.4 & 16.9 & 16.3 & 12.3 & 10.4 & 6.2 & 4.0 & 0.0 & 1.6 & 1.9 \\
\hline Slovene (sl) & 15.0 & 10.9 & 8.1 & 7.3 & 5.9 & 7.2 & 4.3 & 9.4 & 3.7 & 28.1 \\
\hline Spanish (es) & 22.8 & 16.9 & 5.1 & 9.0 & 7.8 & 8.7 & 2.8 & 8.0 & 2.0 & 17.0 \\
\hline Swedish (sv) & 19.3 & 19.5 & 6.9 & 9.3 & 10.8 & 6.4 & 3.9 & 2.5 & 2.7 & 18.8 \\
\hline Tamil (ta) & 27.7 & 0.0 & 9.7 & 3.0 & 7.3 & 6.0 & 1.6 & 6.3 & 2.8 & 35.6 \\
\hline Telugu (te) & 7.3 & 21.3 & 19.5 & 0.0 & 19.2 & 25.6 & 3.5 & 0.1 & 0.0 & 3.6 \\
\hline Turkish (tr) & 38.5 & 8.0 & 10.8 & 1.9 & 6.9 & 9.5 & 3.8 & 0.0 & 1.4 & 19.2 \\
\hline Average & 26.2 & 13.9 & 8.9 & 10.3 & 7.6 & 6.3 & 3.3 & 2.8 & 1.8 & 18.8 \\
\hline
\end{tabular}

Table II.: Selected types of dependency relations and their relative frequency in the harmonized treebanks. One can see repeated patterns in the table such as the dominance of adverbials and attributes, or the relatively stable proportion of subjects. However, the numbers are still biased by imperfections in the conversion procedures (e.g., unrecognized AuxV in certain languages). The abbreviations are inherited from the PDT: Atr $=$ attribute, Adv $=$ adverbial, $\mathrm{Obj}=$ object, $\mathrm{AuxP}=$ preposition, $\mathrm{Sb}=$ subject, Pred $=$ predicate, Coord $=$ coordinating conjunction, AuxV $=$ auxiliary verb, AuxC = subordinating conjunction.

8 They are approximately the same as the dependency relation labels in the Czech CoNLL data set. To illustrate the mapping, more details on [bn] and [en] conversion are presented in Tables IV and V in Appendix B. 
Occasionally the original structure and dependency labels are not enough to determine the normalized output. For instance, the German label RC is assigned to all dependencies that attach a subordinate clause to its parent. The set of HamleDT v1.5 labels distinguishes clauses that act as nominal attributes (Atr) from those that substitute adverbial modifiers (Adv). We look at the part of speech of the parent: if it is a noun, we label the dependency Atr; if it is a verb, we label it Adv. ${ }^{9}$ Thus we also consider the part of speech, the word form, or even further morphological properties. Since the morphological (partof-speech) tagsets also vary greatly across treebanks, we use the Interset approach described by Zeman (2008) to access all morphological information. Interset is a kind of interlingua for parts of speech and morphosyntactic features. Its aim is to provide a unified representation for as many feature values in existing tagsets as possible. We created converters ("drivers") to Interset from all treebank tagsets for which it had not already been available. The normalized treebanks thus provide Interset-unified morphology as well.

In a typical scenario, the harmonization steps are ordered as follows:

1. file format conversion (from various proprietary formats to a commonschema XML) and character encoding conversion (to UTF-8),

2. conversion of morphological tags to the Interset tagset,

3. conversion of dependency relation labels to the set of HamleDT labels,

4. conversion of coordination structures into the HamleDT style (i.e., distinguishing members of coordination and shared modifiers, and attaching them to the main coordination conjunction),

5. other changes in the tree structure (i.e., rehanging nodes to make the dependent-governor relations comply with the HamleDT conventions, including relation orientation) and possibly further refinements of the dependency labels.

The last two points (tree transformations) represent the main focus of the present study; many detailed examples are provided in Section 5.

The implementation of file format converters is relatively straightforward, even though reverse engineering is sometimes needed due to missing technical documentation.

\footnotetext{
${ }^{9}$ Ideally we would also want to distinguish objects $(\mathrm{Obj})$ from adverbials. Unfortunately, this particular source annotation does not provide enough information to make such a distinction.
} 
When implementing the Interset converters, around 200-500 lines of Perl code are typically needed; the code is usually not very challenging from the algorithmic point of view, but requires a very good insight into the annotation guidelines of the respective resource.

Mapping of dependency labels is usually relatively simple to implement too: sometimes it is enough just to recode the original label (e.g. Subj to $\mathrm{Sb}$ ), sometimes the decision must be conditioned by the POS value of the node or of its parent, sometimes the rules are conditioned lexically or by certain structural properties of the tree. However, it all can be done relatively reliably.

More or less the same holds for rehanging the nodes in the fifth step. Typically, there are just a few dozens of transformation rules needed for the third and fifth step (i.e., around 200 lines of Perl code).

The algorithmically most complex step in the harmonization is typically a proper treatment of coordination structures because resolving a coordination structure affects at least three nodes in most cases, coordinations can be nested, and they can combine with almost any dependency relation type. In addition, there are multiple different encodings of coordination structures used in treebanks (17 in HamleDT v1.5), as analyzed in depth by Popel et al. (2013).

Performing the normalization of coordination structures before the normalization of other relations brings about an important advantage: in step 5 , it is possible to work with dependent-governor pairs of nodes in the sense of dependency (not just with child-parent node pairs as stored in the trees), disregarding whether the former or the latter (or both) are coordinated. Without this abstraction, even simple operations, such as swapping the relation orientation between nouns and prepositions, would become quite cumbersome, as one would have to keep all possible combinations in mind, e.g. "with A and B", "with A and with B", "with A and B or with C and D", "with or without A", etc. For more details, please refer to concrete examples in Section 5.

\section{Annotation Styles for Various Phenomena}

In this section, we present a selection of phenomena that we observed and, to various degrees for various languages, included in our normalization scenario. Language codes in brackets give examples of treebanks where the particular approach is employed. The symbol in figure captions marks artificial examples. Figures not marked with contain genuine examples found in real data, though some of them have been shortened. 


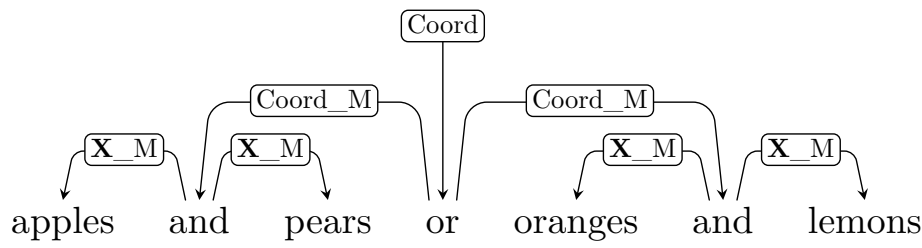

Figure 1. whole structure to its parent. _M denotes members of coordination, i.e., conjuncts.

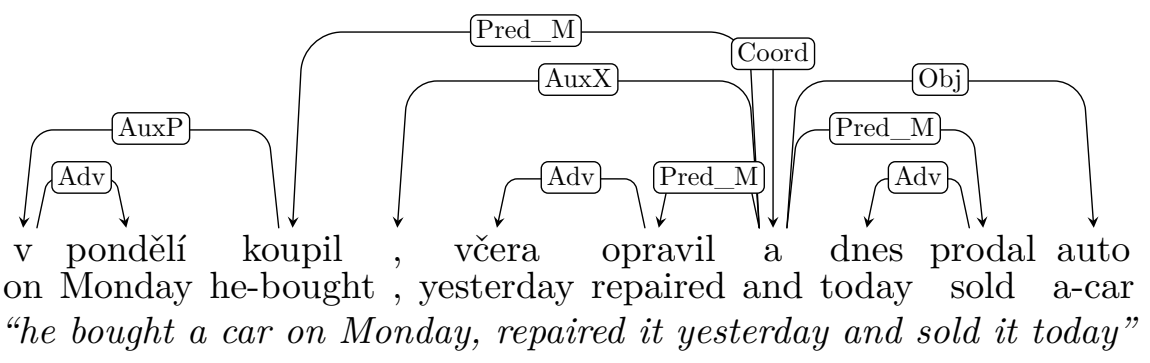

Figure 2. Shared and private modifiers in the Prague style [cs]: Car (auto) is an object shared by all three verbs while the adverbials (on Monday, yesterday, today) are private. The whole structure is in the predicate relation to its parent (which is probably the sentence root), so using the notation of Figure 1: $\mathrm{X}=$ Pred.

Dependency relation labels from the original treebanks that appear in figures are briefly explained in Appendix C.

\subsection{Coordination}

Capturing coordination in a dependency framework has been repeatedly described as difficult for both treebank designers and parsers (and it is generally regarded as an inherent difficulty of dependency syntax as such). Our analysis revealed four families of approaches, which may further vary in the attachment of punctuation, shared modifiers, etc.:

- Prague (Figures 1, 2 and 8). All conjuncts are headed by the conjunction. Used in [ar, bn, cs, el, en, eu, grc, hi, la, nl, sl, ta, te] (Hajič et al., 2006).

- Mel'čukian (Figure 3). The first/last conjunct is the head, others are organized in a chain. Used in [de, ja, ru, sv, tr] (Mel'čuk, 1988).

- Stanford (Figure 4). The first/last conjunct is the head, others are attached directly to it. Used in [bg, ca, es, fi, it, pt] (de Marneffe and Manning, 2008). And 


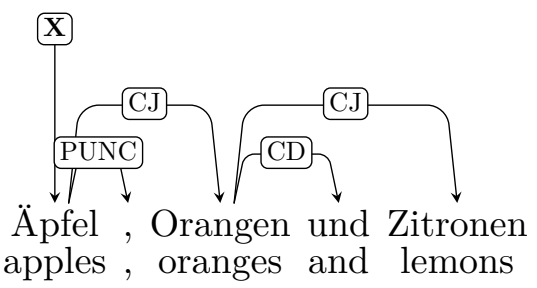

Figure 3. Coordination in the Mel'čukian style as seen in [de].

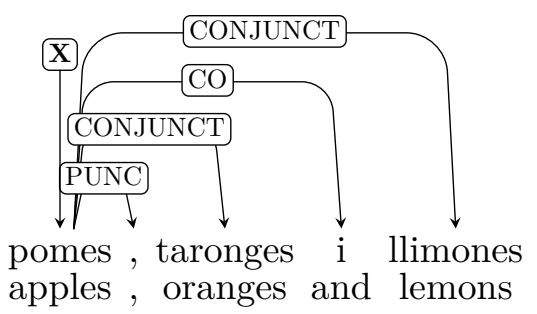

Figure 4. Coordination in the Stanford style as seen in [ca].

- Tesnièrian (Figure 5). There is no common head, all conjuncts are attached directly to the node modified by the coordination structure. Used in [hu] (Tesnière, 1959).

Furthermore, the Prague style provides for nested coordinations, as in "apples and pears or oranges and lemons" (see Figure 1). The asymmetric treatment of conjuncts in the other styles makes nested coordination difficult to read or even impossible to capture in some situations. The Prague style also distinguishes between shared modifiers, such as the subject in "Mary came and cried", from private modifiers of the conjuncts, as in "John came and Mary cried" (see Figure 2). Because this distinction is missing in non-Prague-style treebanks, we cannot recover it reliably. We apply several heuristics, but in most cases, the modifiers of the head conjunct are classified as private modifiers.

Danish (Figure 6) employs a mixture of the Stanford and Mel'čukian styles where the last conjunct is attached indirectly via the conjunction. The Romanian and Russian treebanks omit punctuation tokens (they do not have corresponding nodes in the trees); in the case of Romanian, this means that coordinations of more than two conjuncts are disconnected (Figure 7).

Given the advantages described above, we decided to use the Prague style (in its [cs] flavor) in our harmonized data. There is just one drawback that we are aware of: Occasionally, there may be no node suitable for the coordination head. Most asyndetic constructions do not pose 


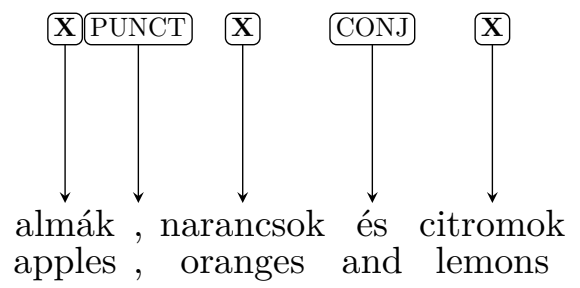

Figure 5. Coordination in the Tesnièrian style as seen in [hu]. All participating nodes are attached directly to the parent of the coordination.

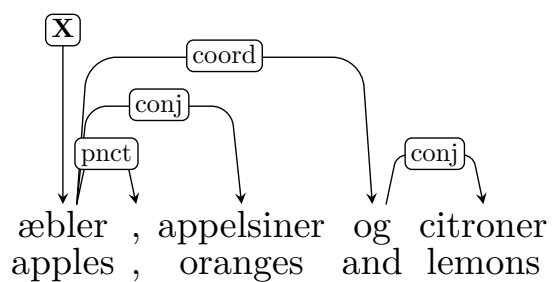

Figure 6. Danish mixture of Stanford and Mel'čukian coordination styles.

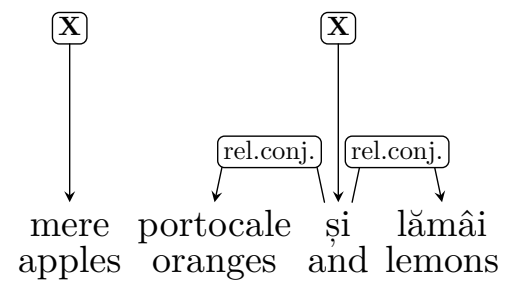

Figure 7. $[\mathrm{ro}$ ] uses Prague coordination style mixed with Tesnièrian because punctuation is missing from data.

a problem because there are commas or other punctuation. Without punctuation, the Prague style would need an extra node - that solution has been adopted by the authors of the [ta] treebank (see Figure 8). Note that one-half of our treebanks already use the Prague style as their native approach, thus they always have a coordination head. In the other half, a fraction of coordinate structures cannot be fully converted (unless we add a new node, which we do not in the current version of HamleDT). For example, 14 out of the 5,988 coordinate structures in [bg] $(0.23 \%)$ lack any conjunction or punctuation that could be made the head. In these cases we currently use the first conjunct instead, effectively backing off to the Stanford style. 


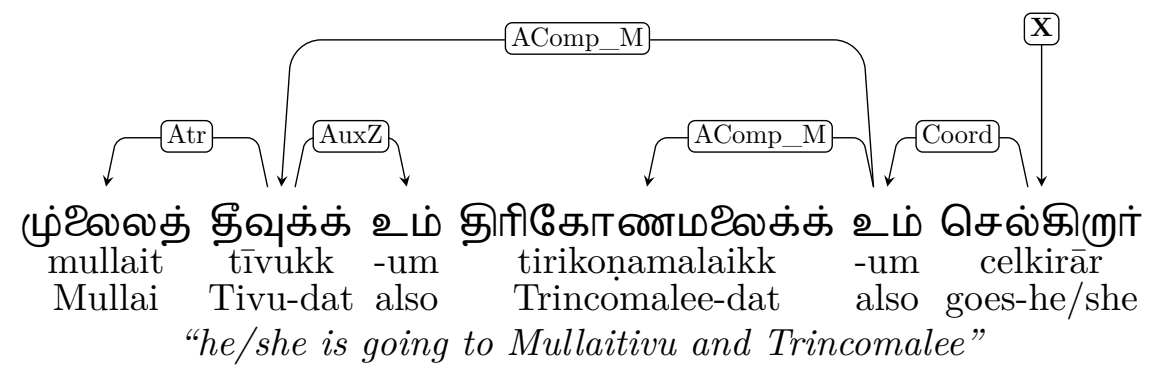

Figure 8. Coordination in [ta]: The coordinating function is performed by the two morphological suffixes -um. They had to be made separate nodes during tokenization because [ta] uses the Prague style and no other coordination head was available except these morphological indicators.

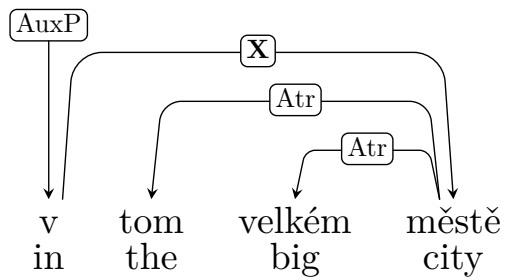

Figure 9. A prepositional phrase in [cs].

\subsection{Prepositions}

Prepositions (or postpositions; Figures 9-11) can either govern their noun phrase (NP) $[\mathrm{cs}$, en, sl, ...] or modify the head of its NP [hi]. When they govern the NP, other modifiers of the main noun are attached either to the noun (in most cases) or to the preposition [de]. The label of the relation of the prepositional phrase to its parent is sometimes found at the preposition [de, en, nl]. Elsewhere, the preposition gets an auxiliary label (such as AuxP in PDT) despite serving as head, and the real label is found at the NP head [cs, sl, ar, el, la, grc].

In HamleDT v1.5 style, prepositions govern their noun phrase because 1. they may govern the form of the noun phrase (e.g. [cs, ru, sl, de]) and 2. this is the approach taken in most of the treebanks we studied. Other modifiers inside the prepositional phrase, such as determiners and adjectives, should depend on the embedded noun phrase. The preposition is labeled with the auxiliary tag AuxP and the real relation between the prepositional phrase and its parent is labeled at the NP head. 


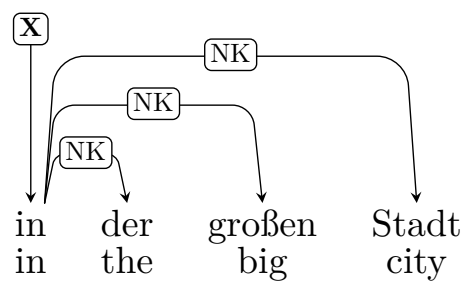

Figure 10. A prepositional phrase in [de].

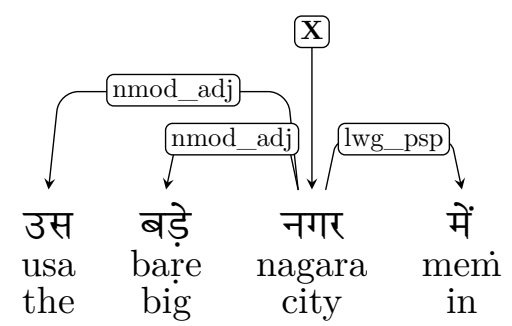

Figure 11.

\subsection{Subordinate Clauses}

There are three main types of subordinate clauses:

- Relative clauses. They modify noun phrases. Typically they are marked by relative pronouns that represent the modified noun and its function within the relative clause. Example: "The man who came yesterday."

- Complement clauses. They serve as arguments of predicates, typically verbs. They are marked by subordinating conjunctions. Example: "The man said that he came yesterday."

- Adverbial clauses. They modify predicates in the same way as adverbs; but they are not selected as arguments. Example: "If the man comes today he will say more."

Roots (predicates) of relative clauses are usually attached to the noun they modify, e.g., in "the man who came yesterday", "came" would be attached to "man" and "who" would be attached to "came" as its subject.

The predicate-modifying clauses use a subordinating conjunction (complementizer, adverbializer) to express their relation to the governing predicate. In treebanks, the conjunction is either attached to the predicate of the subordinate clause [es, ca, pt, de, ro] (Figure 12) or it lies between the embedded clause and the main predicate it modifies 
$\mathrm{X}$

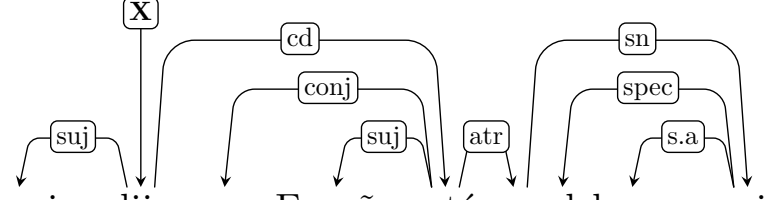

Couceiro dijo que España está en el buen camino

Couceiro said that Spain is in a fair way

Figure 12. Subordinate clause in [es].

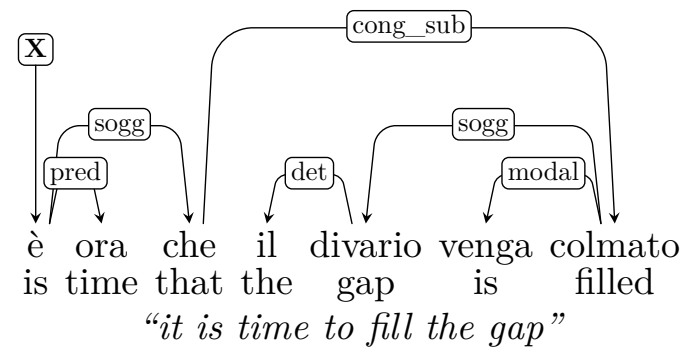

Figure 13. Subordinate clause in [it].

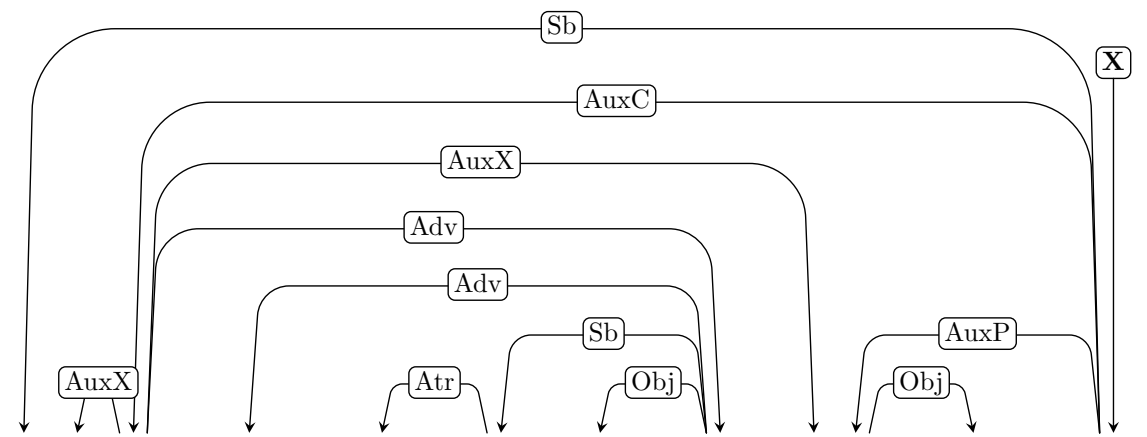

ipse, cum primum pabuli copia esse inciperet, ad exercitum venit

he , as soon forage plenty be began, to army came "as soon as there began to be plenty of forage, he himself came to the army"

Figure 14. Subordinate clause in [la].

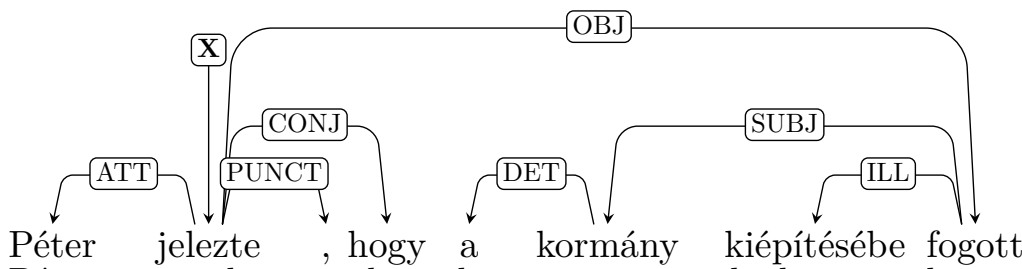

Péter pointed-out, that the government deployment began

Figure 15. Subordinate clause in [hu]. 


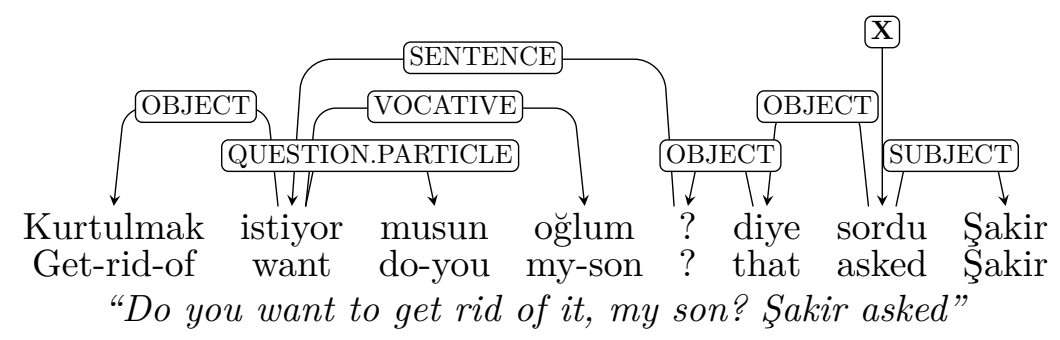

Figure 16. Subordinate clause (direct speech) in [tr]: Here, the question mark serves as the head of the direct question.

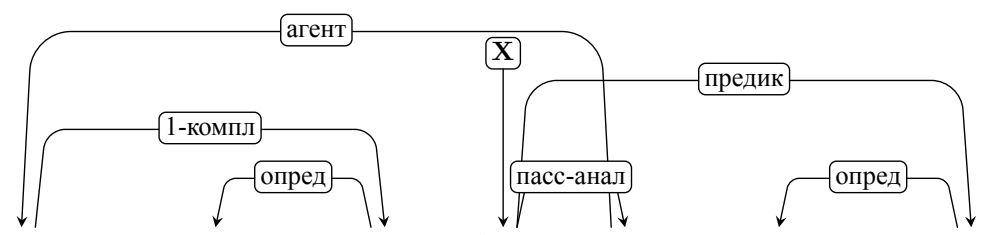

народами Урартского царства была создана высокая цивилизация narodami Urartskogo carstva byla sozdana vysokaja civilizacija by-nations of-Urartu empire was created high civilization "a high civilization was created by the nations of Urartu"

Figure 17. Passive construction in [ru]: Finite auxiliary verb (была) is the head, passive participle (создана) depends on it. As a result, the agent (народами) is attached non-projectively to the participle (создана).

[cs, en, hi, it, la, ru, sl] (Figure 13). In the latter case, the label of the relation of the subordinate clause to its parent can be assigned to the conjunction [en, hi, it] or to the clausal predicate [cs, la, sl] (Figure 14). The comma before the conjunction is attached either to the conjunction or to the subordinate predicate.

The subordinating conjunction may also be attached as a sibling of the subordinate clause [hu], an analogy to the Tesnièrian coordination style (Figure 15). In Figure 16, a direct question in [tr] is rooted by the question mark, which is attached to a subordinating postposition.

The Romanian treebank is segmented into clauses instead of sentences, so every clause has its own tree, and inter-clausal relations are not annotated.

HamleDT v1.5 style follows the [cs, sl, la] approach to subordinate clauses (see Figures 14 and 19).

\subsection{Verb Groups}

Various sorts of verbal groups include analytical verb forms (such as auxiliary + participle), modal verbs with infinitives, and similar constructions. Dependency relations, both internal (between group ele- 


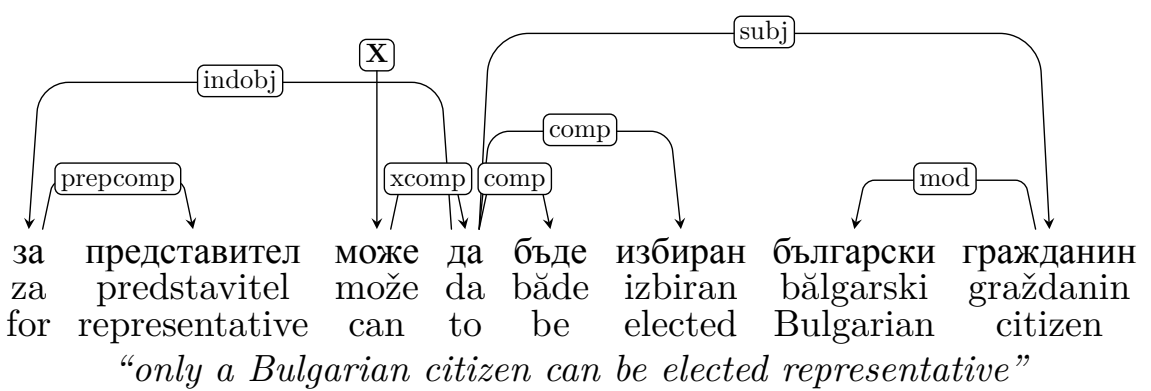

Figure 18. Modal passive construction in [bg]: The finite modal verb (може) is the head, the infinitive particle $(\partial a)$ is the second-level head. The infinitive auxiliary ( $(б ъ \partial e)$ is attached to $\partial a$, as is the passive participle of the content verb (избиран) and the two arguments of the content verb, one of them (за представител) non-projectively.

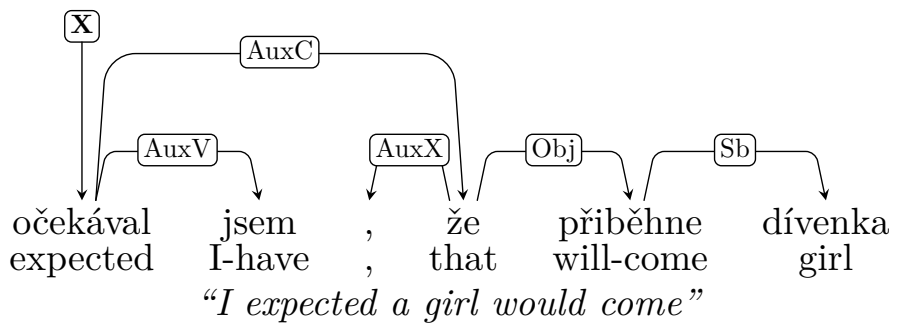

Figure 19. Past tense in [cs]: The participle of the content verb (očekával) governs the finite form of the auxiliary (jsem). Making the auxiliary the head would cause problems because it is not always present, e.g., omitting it in this sentence would just shift the sentence to the 3rd person meaning ("He expected a girl would come.")

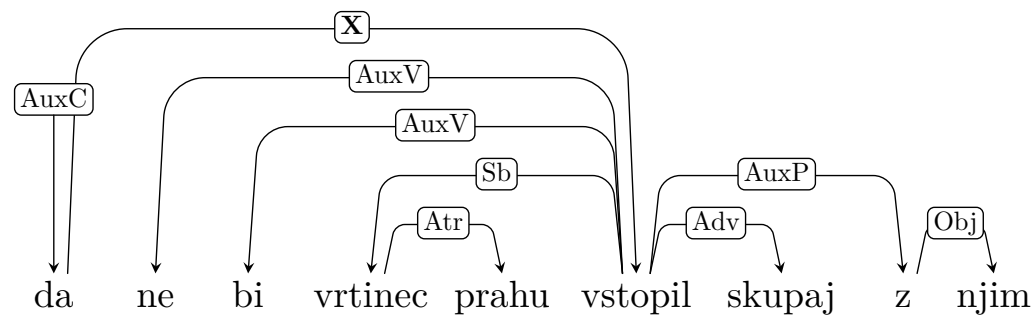
so-that not would vortex of-dust entered together with him "so that the vortex of dust would not enter together with him"

Figure 20. Negated conditional construction in [sl]. The past participle of the content verb (vstopil) is the head, the negative particle (ne) and the auxiliary (bi) depend on it.

ments) and external (leading to the parent on the one side and verb modifiers on the other side), may be defined according to various criteria: content verb vs. auxiliary, finite form vs. infinitive, or subject- 


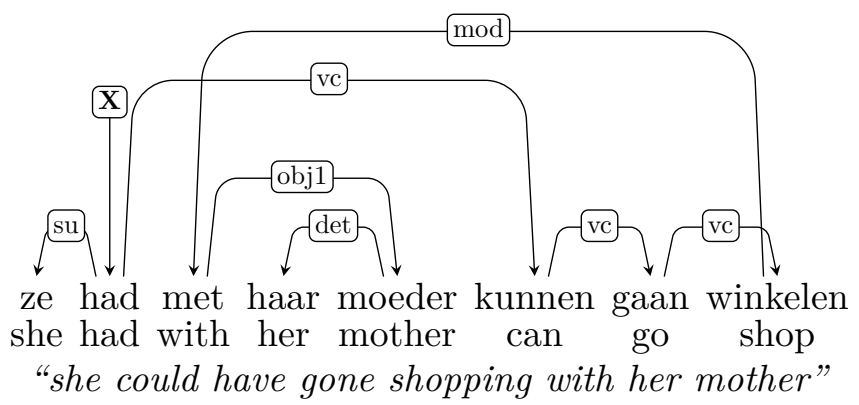

Figure 21. Past modal construction in [nl]. The finite auxiliary verb (had) is the head. The subject (ze) is attached to the finite verb (had) while the modifier (met haar moeder) is attached non-projectively to the content verb (winkelen).

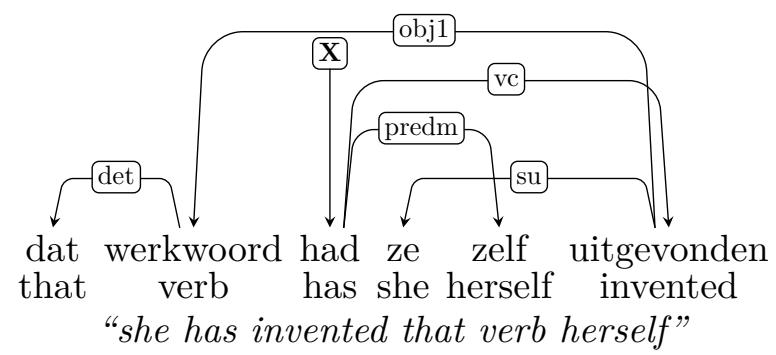

Figure 22. Another example from [nl]. Unlike in other treebanks, even the subject $(z e)$ is attached to the non-head participle (uitgevonden).

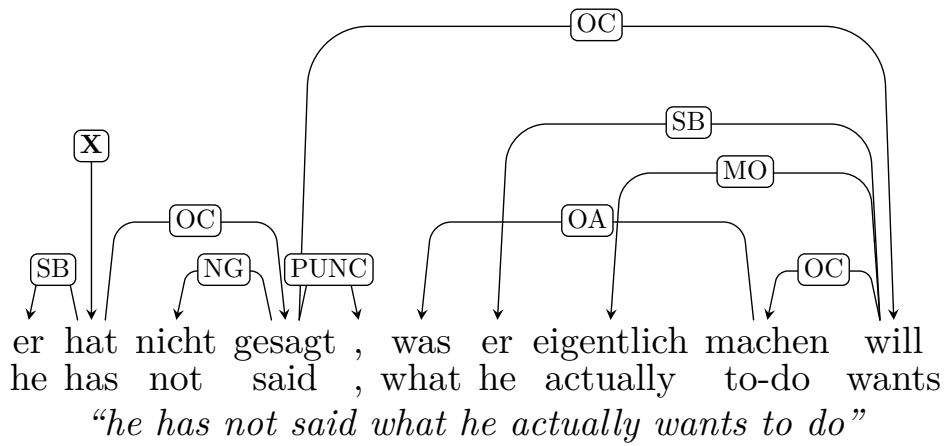

Figure 23. A combination of perfect tense, modal verb, and infinitive in [de]. Infinitives are attached to modals as their objects in many treebanks, including [de]. The finite auxiliary verb (hat) is the head of the perfect tense, the participle (gesagt) depends on it. The subject (er) is attached to the finite verb (hat) while the object clause (was er eigentlich machen will) is attached to the content verb (gesagt).

verb agreement, which typically holds for finite verbs, sometimes for participles but not for infinitives. 


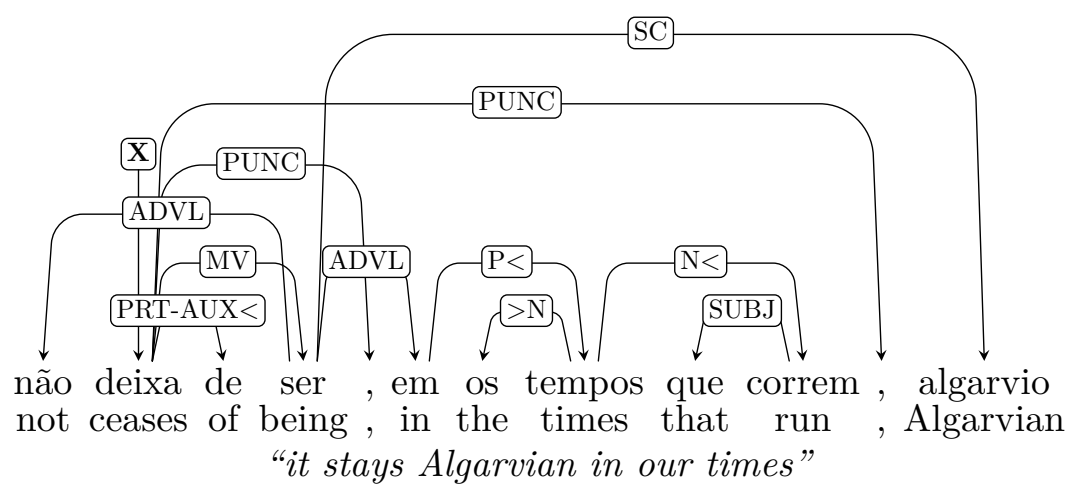

Figure 24. Infinitive with preposition in [pt]: Preposition (de) is not attached between the phase verb (deixa) and the infinitive (ser). The negative particle (não) is attached non-projectively to the non-head verb (ser). Moreover, the commas around the parenthetical (em os tempos que correm) are also non-projective.

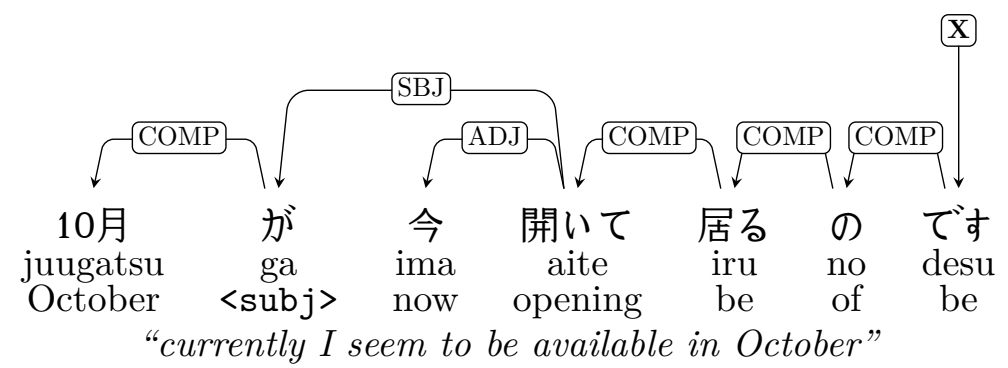

Figure 25. [ja] Desu is the polite copula. Aite is the conjunctive form of aku= "to open". The auxiliary iru with conjunctive of content verb together form the progressive tense. Japanese is an SOV language and left-branching structures are much preferred.

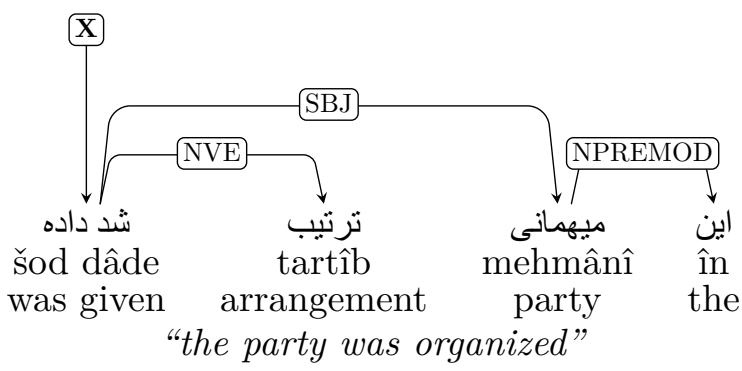

Figure 26. [fa] Note that the dependency tree of the sentence (În mehmânî tartîb šod dâde.) is ordered right-to-left, the way Persian is written. The analytical passive šod dâde is represented by a single node (token).

Participles often govern auxiliaries [es, ca, it, ro, sl] (Figures 19 and 20); elsewhere the finite verb is the head [pt, de, nl, en, sv, ru] 


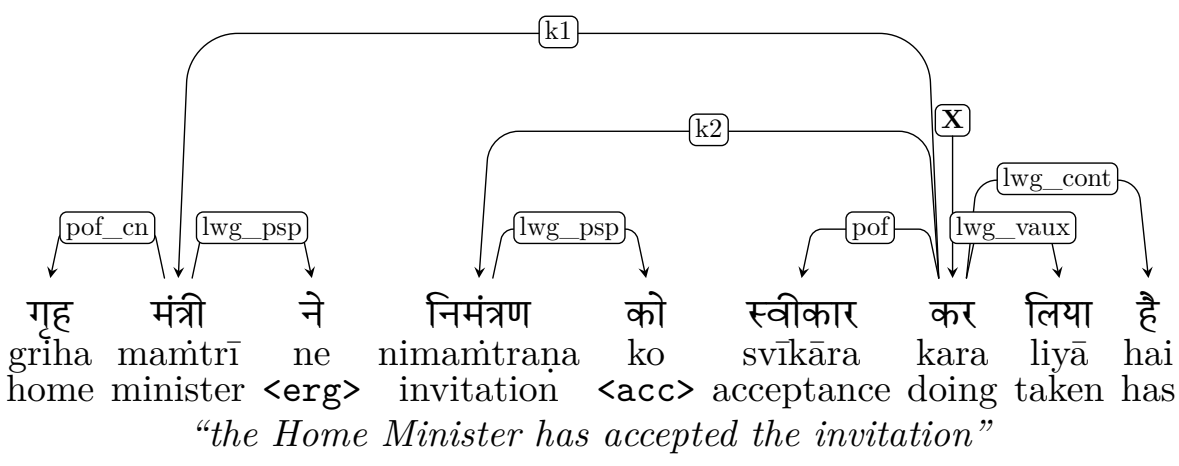

Figure 27. [hi] Kara is a light verb stem, svīkāra karanā means "to accept". Liyā, the perfect participle of len $\bar{a}$ "to take", is another light verb, specifying the direction of the result of the action. Hai is the auxiliary verb "to be" in finite form. Content verbs govern verbal groups in the [hi] treebank; as the main verb in this case is a compound verb (svikāra kara), the head node of the two (kara) governs the whole group, even though the real content lies in the nominal element (svikkarra).

(Figures 17, 22 and 23), and finally, [cs] mixes both approaches based on semantic criteria. In [hi, ta], the content verb, which could be a participle or a bare verb stem, is the head, and auxiliaries (finite or participles) are attached to it (Figure 27).

The head typically holds the label describing the relation of the whole verbal group to its parent. As for child nodes, subjects and negative particles are often attached to the head, especially if it is the finite element [de, en], while the arguments (objects) are attached to the content element whose valency slot they fill (often participle or infinitive). Sometimes even the subject (in [nl]) or the negative particle (in [pt]) can be attached to the non-head content element (Figure 22). Various infinitive-marking particles (English "to", Swedish "att", Bulgarian " $\partial a$ ") are usually treated similarly to subordinating conjunctions, i.e., they either govern the infinitive [en, da, bg] or are attached to it [de, sv]. In [pt], prepositions used between the main verb and the infinitive ("estâo a usufruir"= "are enjoying") are attached to the finite verb (Figure 24). In [bg], all modifiers of the verb including the subject are attached to the infinitive particle " $\partial a$ " instead of the verb below it (Figure 18).

We intend to unify verbal groups under a common approach, but the current version 1.5 of HamleDT does not do so yet. This part is more language-dependent than the others and a further analysis is needed. 

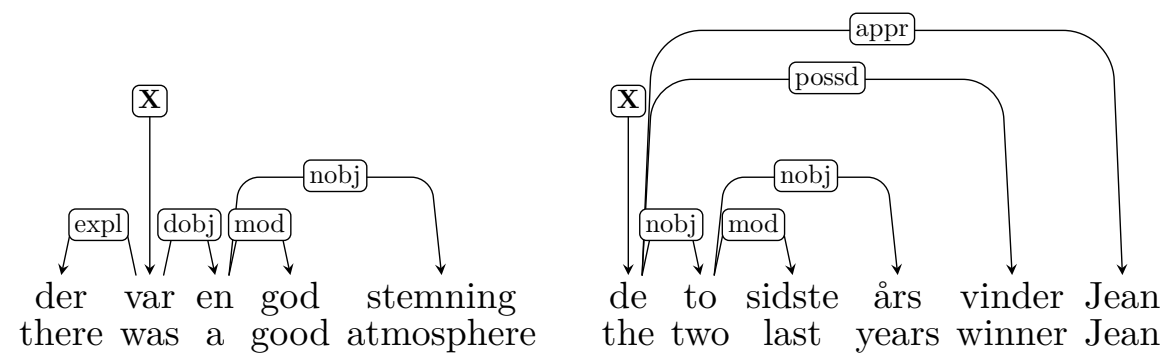

Figure 28. Two fragments from [da] show determiners and numerals governing noun phrases.

\subsection{Determiner Heads}

The Danish treebank is probably the most extraordinary one. Nouns often depend on determiners, numerals, etc. (see Figure 28). This approach is very rare in dependency treebanks, although it has its advocates among linguists (Hudson, 2004; Hudson, 2010). ${ }^{10}$

In HamleDT v1.5, we attach articles as well as other determiners to their nouns and numerals to the counted nouns. ${ }^{11}$

\subsection{Punctuation}

Table III presents an overview of punctuation treatment in the treebanks. Details and exceptions are discussed below. The type codes at paragraph beginnings refer to the columns of the table.

Pair/Pcom: Paired punctuation marks (quotation marks, brackets, parenthesizing commas (Pcom) or dashes) are typically attached to the head of the segment between them. Occasionally, they are attached one level higher, to the parent of the enclosed segment, or even higher, if the parent is member of a verbal group. Attaching punctuation to higher levels may break projectivity, as in Figure 24. The [pt] approach attaches paired punctuation to the parent of the interior segment (i.e. to the parent of the head of the segment, not to the head), unless the parent is the root or there are tokens outside the punctuation that depend on the head inside. In this latter case, the punctuation is attached to the inner head. In [tr], the Pcom column does not necessarily refer to paired punctuation; some commas are just attached to the root, which may result in non-projectivity.

\footnotetext{
10 In Chomskian (constituency-based) approaches, it is the standard analysis that determiners function as the head of a noun phrase.

11 Note however that numerals governing nouns are not restricted to [da]. Czech has a complex set of rules for numerals (motivated by the morphological agreement), which may result under some circumstances in the numeral serving as the head.
} 
Rcom: Similarly, commas before and after a relative clause are typically attached either to the root of the relative clause (be it verb or conjunction) or to its parent. In [la], the clause is sometimes headed by a subordinating conjunction, but the comma is attached to the verb below. Note, however, that a comma terminating a clause may have multiple functions: it may at the same time delimit several nested clauses, a parenthetical phrase, and/or a conjunct.

In several languages, commas (in [fa]) or all punctuation symbols (in $[\mathrm{eu}, \mathrm{it}, \mathrm{nl}]$ ) are systematically attached to neighboring tokens.

Coord: Commas, semicolons, or dashes can also substitute coordinating conjunctions, which is important especially if the Prague style of coordination is used (see Section 5.1). In [te], this is the sole function of commas (see Figure 29). In [da], which does not follow the Prague approach to coordination, we observed two adjectives modifying the same noun, separated by a comma; the comma was attached to the first "conjunct". We list the case in the Coord column although the structure was not formally tagged as coordination. In [hu], coordinating commas are normally attached to the parent of the coordination. Parents that are roots of the tree are an exception: in such cases, the comma is used as the head of the coordination.

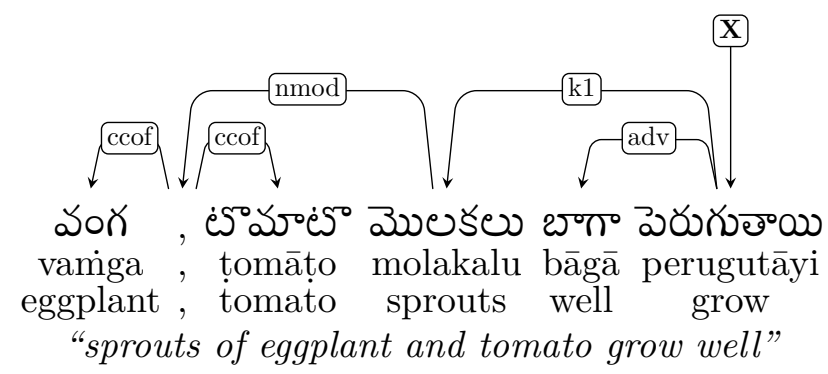

Figure 29. Coordinating comma in [te].

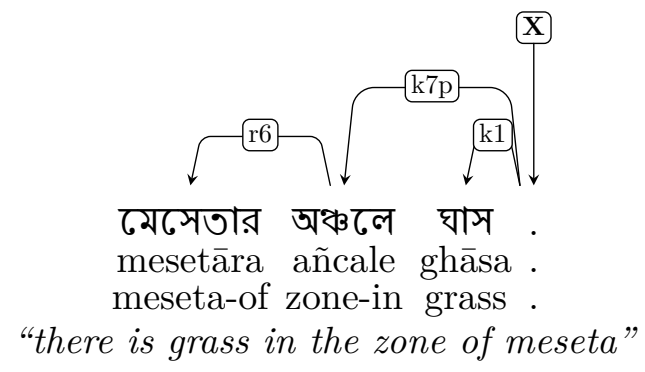

Figure 30. NULL-like usage of period in [bn]. The node with the period represents a dropped copula. Elsewhere in the treebank, such nodes are labeled by the pseudo-word-form "NULL". 


\begin{tabular}{|c|c|c|c|c|c|c|c|}
\hline Language & Fin & Pair & Pcom & Rcom & Coord & Coor1 & Apos \\
\hline Arabic (ar) & RN & SH & SH & & $\mathrm{HD}$ & $\overline{(\mathrm{PT})}$ & \\
\hline Basque (eu) & $\mathrm{PT}$ & $\overline{\mathrm{PT}}$ & $\overline{\mathrm{PT}}$ & $\mathrm{PT}$ & & $\mathrm{PT}$ & $\mathrm{PT}$ \\
\hline Bengali (bn) & $\left(\mathrm{MP}^{*}\right)$ & & & & HD & & \\
\hline Bulgarian (bg) & MP & $\mathrm{SH}$ & $\mathrm{SH}$ & $\mathrm{SH}$ & $\mathrm{SH}$ & $\mathrm{SH}$ & $\mathrm{SH}$ \\
\hline Catalan (ca) & MP & $\mathrm{SH}$ & $\mathrm{SH}$ & $\mathrm{SH}$ & $\mathrm{SH}$ & $\mathrm{SH}$ & $\mathrm{SH}$ \\
\hline Czech (cs) & $\mathrm{RN}$ & $\mathrm{SH}$ & $\overline{\mathrm{SH}}$ & $\mathrm{SH}$ & HD & $\mathrm{SH}$ & $\mathrm{HD}$ \\
\hline Danish (da) & MP & $\mathrm{SH}$ & & $\mathrm{SP} / \mathrm{SH}$ & $\mathrm{PT}^{*}$ & $\mathrm{SH}$ & \\
\hline Dutch (nl) & PW & PW & PW & PW & & $\mathrm{PW}$ & PW \\
\hline English (en) & MP & SH? & $\mathrm{SP}$ & SP & $\mathrm{HD}$ & $\mathrm{SH}$ & SP \\
\hline Estonian (et) & MP & $\mathrm{SH} \mid \mathrm{SP}$ & $\mathrm{SP}$ & $\mathrm{SH} \mid \mathrm{SP}$ & $\mathrm{HD}$ & $\mathrm{SH}$ & SP \\
\hline Finnish (fi) & MP & SH & $\mathrm{SH}$ & $\mathrm{SH}$ & $\mathrm{SH}$ & $\mathrm{SH}$ & \\
\hline German (de) & MP & SH? & SP & SP & $\mathrm{SH}$ & $\mathrm{PC}$ & \\
\hline Greek (el) & $\mathrm{RN}$ & SH & & & $\mathrm{HD}$ & $\mathrm{SH}$ & HD \\
\hline Greek (grc) & $\mathrm{RN}$ & $(\mathrm{SH})$ & SH & SH & $\mathrm{HD}$ & $\mathrm{SH}$ & $\mathrm{HD}$ \\
\hline Hindi (hi) & MP & $\mathrm{SH}$ & & $(\mathrm{SP})$ & & $\mathrm{PC} \mid \mathrm{PT}$ & \\
\hline Hungarian (hu) & MP & $\mathrm{SH} \mid \mathrm{SP}$ & $\mathrm{SH} \mid \mathrm{SP}$ & $\mathrm{SP}$ & $\mathrm{HD} \mid \mathrm{SP}^{*}$ & SP & \\
\hline Italian (it) & $\mathrm{PT}$ & $\mathrm{NT} / \mathrm{PT}$ & PT & $\mathrm{PT}$ & & $\mathrm{PT}$ & $\mathrm{PT}$ \\
\hline Japanese (ja) & $\mathrm{MP}^{*}$ & & & & & & \\
\hline Latin (la) & & & $\mathrm{SH}$ & $\mathrm{SH}^{*}$ & HD & $\mathrm{SH}$ & HD \\
\hline Persian (fa) & MP & $\mathrm{SH}$ & $\mathrm{PT}$ & $\mathrm{PT}$ & & $\mathrm{PT}$ & $\mathrm{PT}$ \\
\hline Portuguese (pt) & MP & $\mathrm{SP}^{*}$ & SP & $\mathrm{SP}$ & SH & $\mathrm{SH}$ & $\mathrm{SP}$ \\
\hline Romanian (ro) & & & & punctuat & & & \\
\hline Russian (ru) & & & no & punctuat & & & \\
\hline Slovene (sl) & $\mathrm{RN}$ & $\mathrm{SH}$ & $\mathrm{SH}$ & SH & $\mathrm{HD}$ & $\mathrm{SH}$ & $\mathrm{HD}$ \\
\hline Spanish (es) & MP & SH & $\mathrm{SH}$ & $\mathrm{SH}$ & $\mathrm{SH}$ & $\mathrm{SH}$ & $\mathrm{SH}$ \\
\hline Swedish (sv) & MP & $\mathrm{NT} / \mathrm{PT}$ & $\mathrm{SP}$ & $\mathrm{SP}$ & $\mathrm{PC}$ & $\mathrm{PC}$ & $\mathrm{SP}$ \\
\hline Tamil (ta) & $\mathrm{RN}$ & $\mathrm{SH}$ & SP & SP & $\mathrm{HD}$ & $\mathrm{SH}$ & \\
\hline Telugu (te) & $(\mathrm{MP})$ & & & & HD & & \\
\hline Turkish (tr) & $\mathrm{RR}$ & & $\mathrm{RN}^{*}$ & $\mathrm{CH}$ & $\mathrm{CH}$ & $\mathrm{CH}$ & \\
\hline HamleDT v1.5 & $\mathrm{RN}$ & $\mathrm{SH}$ & $\mathrm{SH}$ & $\mathrm{SH}$ & HD & $\mathrm{SH}$ & $\mathrm{SH}$ \\
\hline
\end{tabular}

Table III.: Punctuation styles overview. $\mathrm{RN}=$ attached to the artificial root node; $\mathrm{RR}=$ attached to the artificial root and serving as root for the rest of the sentence, i.e., heading the main predicate; MP = attached to the main predicate; NT $=$ attached to the next token; PT $=$ attached to the previous token; $\mathrm{PW}=$ attached to the previous word (i.e., non-punctuation token); $\mathrm{PC}=$ attached to the previous conjunct; $\mathrm{SH}=$ attached to the head of the rel. clause / subtree inside paired punc. / coordination / second appos. member; SP $=$ attached to the (grand)parent node of the rel. clause / subtree inside paired punc.; or to the first appos. member; $\mathrm{CH}=$ chain: attached to parent, and the head of the clause attached to the comma; for Coord, previous conjunct attached to comma, comma attached to next conjunct; HD = serving as head of coordination; $(X)=$ rare in this treebank, based on very few observations; $X / Y=$ initial $X$, final $Y ; X \mid Y=$ both observed; $X$ ? = unexplained exceptions observed; $X^{*}=$ see text for more details; empty cell $=$ not observed. 
Coor1: Multi-conjunct coordination often involves one conjunction and one or more commas. Even within the same coordination family, multiple attachment schemes are possible for the commas (the previous conjunct, the head of the coordination, etc.) Additional commas are rare in [ar], where repeated conjunctions are more common.

Apos: Constructions in which two phrases describe the same object are called appositions. These are mostly but not solely noun phrases separated by a comma, dash, bracket, etc. as in "Nicoletta Calzolari, the chief editor". Appositions are treated in the same way as parenthesis in most treebanks - the second phrase is attached to the first. Other treebanks regard appositions as coordinations - the punctuation serves as the head, with both phrases attached symmetrically.

Fin: Sentence-final punctuation (period, question mark, exclamation mark, three dots, semicolon, or colon) is attached to the artificial root node [cs, ar, sl, grc, ta], to the main predicate [bg, ca, da, de, en, es, et, fi, hu, pt, sv], or to the previous token [eu, it, ja, nl]. ${ }^{12}$ In [la, ro, $\mathrm{ru}$ ], there is no final punctuation. It is also extremely rare in [bn, te]; however, there are a few punctuation nodes in [bn] that govern other nodes in the sentence. In fact, these nodes actually should have been labeled NULL to represent a copula or other constituents missing from the surface (Figure 30). Such NULL nodes appear elsewhere in [bn]. Punctuation is attached to the artificial root node in [tr] but instead of being a sibling of the main predicate, it governs the predicate. Note that some languages (e.g. Czech) may require final quotation marks (if present) to appear after the final period, but in [cs], it is not treated as final punctuation (unlike the period). Such quotation marks may end up attached non-projectively to the main verb.

A few treebanks [bg, cs, la, sl] use separate nodes for periods that mark abbreviations and ordinal numbers. These nodes are attached to the previous node (i.e., the abbreviation). In [cs], this rule has a higher priority even in cases where a period serves as an abbreviation marker and a sentence terminator at the same time. Most other treebanks are tokenized so that the period shares a node with the abbreviation (see also Section 5.7).

In HamleDT v1.5, we treat apposition as parenthesis, we attach paired punctuation to the root of the subtree inside and sentence-final punctuation to the artificial root node, mostly for consistency reasons. For the other punctuation types, a further analysis is needed.

\footnotetext{
12 In [ja], the previous token essentially means the main predicate, but if it is followed by a question particle then the punctuation node is attached to the particle.
} 


\subsection{Tokenization and Sentence Segmentation}

The only aspect that remains unchanged in HamleDT is tokenization and segmentation. Our harmonized trees always have the same number of nodes and sentences as the original annotation, despite some variability in the approaches we observe in the original treebanks.

\section{Multi-word expressions and missing tokens}

Some treebanks collapse multi-word expressions into single nodes [ca, da, es, eu, fa, hu, it, nl, pt, ro, ru]. Collapsing is restricted to personal names in [hu] and to named entities in [ro]. In [fa], it is used for analytical verb forms. The word form of the node is composed of all the participating words, joined by underscore characters or even by spaces [fa].

In [bn, te], dependencies are annotated between chunks instead of words (Figure 31). Therefore, one node may represent a whole noun phrase with modifiers and postpositions. The treebank only shows chunk headwords, which means we cannot reconstruct the original sentence. On a similar note, punctuation tokens have been deleted from two treebanks ([ro, ru]; see also Section 5.6).

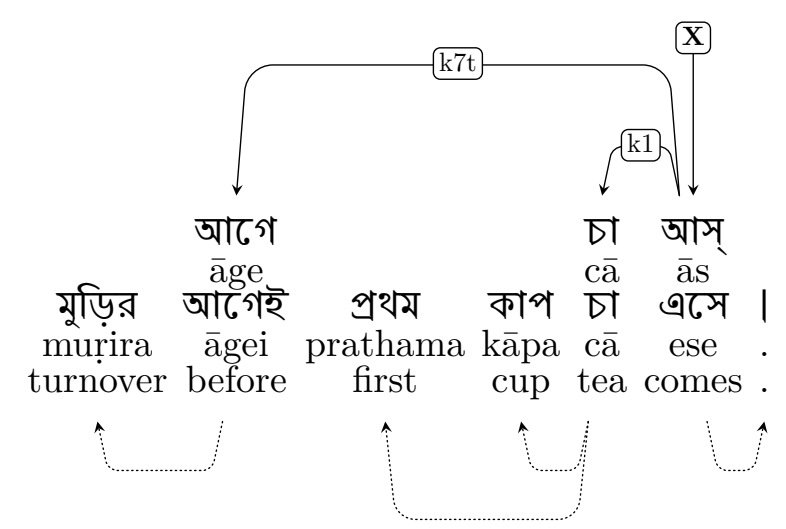

Figure 31. "The first cup of tea comes before the turnover." [bn] captures dependencies between chunks, not between tokens. Every sentence has been chunked and chunk headwords serve as nodes of the tree (their word forms are replaced by lemmas). The dotted dependencies below the sentence indicate which tokens belong to which chunk. Neither these dependencies nor the chunk-dependent words are visible in the treebank. The original sentences cannot be reconstructed from the trees.

\section{Split tokens}

On the other hand, orthographic words may be split into syntactically autonomous parts in some treebanks [ar, fa]. For instance, the Arabic 
word وبالفالوجة (wabiālfālüjah = "and in al-Falujah") is separated into $w a /$ CONJ $+b i /$ PREP + AlfAlwjp/NOUN_PROP. In [ta], the suffix -um indicating a coordination is treated as a separate token (see Section 5.1 and Figure 8).

\section{Artificial nodes}

Occasionally [bn, hi, te, ru], we see an inserted NULL node, which mostly stands for participants deleted on the surface, e.g., copulas [bn, ru] or conjuncts as in the Hindi example in Figure 32.

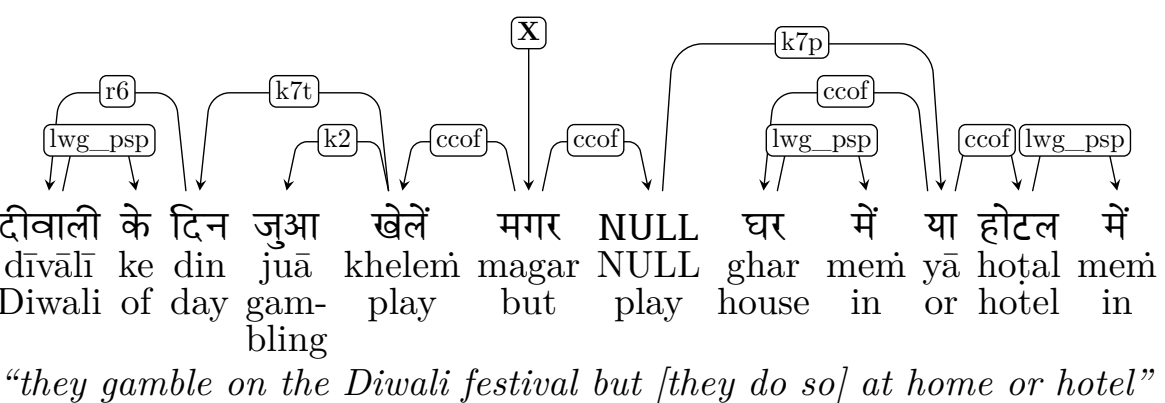

Figure 32. A NULL node for a deleted verb (serving as head of conjunct) in [hi].

Along the same lines, some treebanks of pro-drop languages [ca, es] use empty nodes (with artificial word "_") representing missing subjects, as in the following Spanish sentence: "_ Afirmó que _ sigue el criterio europeo y que _ trata de incentivar el mercado donde no lo hay." = "He said he follows the European standard and encourages the market where there is none." All the underscores mark subjects of the following verbs and could be translated as "he".

Underscore/NULL nodes also appear in [tr], where they encode additional information related to morphological derivation.

\section{Sentence segmentation}

Similarly to tokenization, we also treat sentence segmentation as fixed, despite some less usual solutions: in [ar], sentence-level units are paragraphs rather than sentences, which explains the high average sentence length in Table I. In contrast, [ro] annotates every clause as a separate tree.

\section{Obtaining HamleDT}

Twelve harmonized treebanks from HamleDT v1.5 [ar, cs, da, fa, fi, grc, la, nl, pt, ro, sv, ta] are directly available for download from our web site: 
http://ufal.mff.cuni.cz/hamledt

The license terms of the rest of the treebanks prevent us from redistributing them directly (in their original or normalized form), but most of them are easily acquirable for research purposes, under the links given in Appendix A). We provide the software that can be used to normalize and display the data after obtaining them from the original provider.

All the normalizations are implemented in Treex (formerly TectoMT) (Popel and Žabokrtský, 2010), a modular open-source framework for structured language processing, written in Perl. ${ }^{13}$ In addition to normalization scripts for each treebank, Treex contains also other transformations, so for example, coordinations in any treebank can be converted from Prague to Stanford style.

The tree editor $\operatorname{TrEd}^{14}$ can open Treex files and display original and normalized trees side-by-side on multiple platforms.

\section{Conclusion}

We provide a thorough analysis and discussion of varying annotation approaches to a number of syntactic phenomena, as they appear in publicly available treebanks, for many languages.

We propose a method for automatic normalization of the discussed annotation styles. The method applies transformation rules conditioned on the original structural annotation, dependency labels and morphosyntactic tags. We also propose unification of the tag sets for parts of speech, morphosyntactic features, and dependency relation labels. We take care to make the structural transformations and the morphosyntactic tagset unification as reversible as possible. ${ }^{15}$

We provide an implementation of the transformations in the Treex NLP framework. Treex can also be used for transforming the data to other annotation styles besides the one we propose (cf. Popel et al. (2013)). The resulting collection of harmonized treebanks, called HamleDT v1.5, is available to the research community according to the original licenses. A subset of the treebanks whose license terms permit redistribution is available directly from us. For the rest, users need to acquire the original data and apply our transformation tool.

Several future directions of our work are possible. Besides deepening the current level of harmonization (especially for verbal groups),

13 http://ufal.mff.cuni.cz/treex/

14 http://ufal.mff.cuni.cz/tred/ with EasyTreex extension

15 We do not attempt at reversibility when unifying dependency relations. 
we plan on adding new treebanks and languages, for which resources exist (e.g., French, Hebrew, Chinese, Icelandic, Ukrainian or Georgian). We also want to run parsing experiments and evaluate the various annotation styles from the point of view of learnability by parsers.

\section{Acknowledgements}

The authors wish to express their gratitude to all the creators and providers of the respective corpora.

The work on this project was supported by the Czech Science Foundation grant no. P406/11/1499 and no. P406/14/06548P, by the European Union Seventh Framework Programme under grant agreement FP7-ICT-2013-10-610516 (QTLeap), and by research resources of the Charles University in Prague (PRVOUK). This work has been using language resources developed and/or stored and/or distributed by the LINDAT/CLARIN project of the Ministry of Education of the Czech Republic (project LM2010013).

Finally, we are very grateful for the numerous valuable comments provided by the anonymous reviewers.

\section{References}

Aduriz, I., M. J. Aranzabe, J. M. Arriola, A. Atutxa, A. Díaz de Ilarraza, A. Garmendia, and M. Oronoz: 2003, 'Construction of a Basque dependency treebank'. In: Proceedings of the 2nd Workshop on Treebanks and Linguistic Theories.

Afonso, S., E. Bick, R. Haber, and D. Santos: 2002, "Floresta sintá(c)tica": a treebank for Portuguese'. In: Proceedings of the 3rd International Conference on Language Resources and Evaluation (LREC). pp. 1968-1703.

Atalay, N. B., K. Oflazer, B. Say, and I. Inst: 2003, 'The Annotation Process in the Turkish Treebank'. In: In Proc. of the 4th Intern. Workshop on Linguistically Interpreteted Corpora (LINC).

Bamman, D. and G. Crane: 2011, 'The Ancient Greek and Latin Dependency Treebanks'. In: C. Sporleder, A. Bosch, and K. Zervanou (eds.): Language Technology for Cultural Heritage, Theory and Applications of Natural Language Processing. Springer Berlin Heidelberg, pp. 79-98.

Bengoetxea, K. and K. Gojenola: 2009, 'Exploring Treebank Transformations in Dependency Parsing'. In: Proceedings of the International Conference RANLP2009. Borovets, Bulgaria, pp. 33-38, Association for Computational Linguistics.

Bharati, A., V. Chaitanya, and R. Sangal: 1994, Natural Language Processing: A Paninian Perspective. New Delhi: Prentice-Hall of India.

Bick, E., H. Uibo, and K. Müürisep: 2004, 'Arborest - a VISL-Style Treebank Derived from an Estonian Constraint Grammar Corpus'. In: Proceedings of Treebanks and Linguistic Theories. 
Boguslavsky, I., S. Grigorieva, N. Grigoriev, L. Kreidlin, and N. Frid: 2000, 'Dependency treebank for Russian: Concept, tools, types of information'. In: Proceedings of the 18th conference on Computational linguistics-Volume 2. pp. 987-991.

Bosco, C., S. Montemagni, A. Mazzei, V. Lombardo, A. Lenci, L. Lesmo, G. Attardi, M. Simi, A. Lavelli, J. Hall, J. Nilsson, and J. Nivre: 2010, 'Comparing the Influence of Different Treebank Annotations on Dependency Parsing'.

Brants, S., S. Dipper, P. Eisenberg, S. Hansen, E. König, W. Lezius, C. Rohrer, G. Smith, and H. Uszkoreit: 2004, 'TIGER: Linguistic Interpretation of a German Corpus'. Journal of Language and Computation 2(4), 597-620. Special Issue.

Buchholz, S. and E. Marsi: 2006, 'CoNLL-X shared task on multilingual dependency parsing'. In: In Proc. of CoNLL. pp. 149-164.

Civit, M., M. A. Martí, and N. Bufí: 2006, 'Cat3LB and Cast3LB: From Constituents to Dependencies.'. In: T. Salakoski, F. Ginter, S. Pyysalo, and T. Pahikkala (eds.): FinTAL, Vol. 4139 of Lecture Notes in Computer Science. pp. 141-152, Springer.

Călăcean, M.: 2008, 'Data-driven Dependency Parsing for Romanian'. Master's thesis, Uppsala University.

Csendes, D., J. Csirik, T. Gyimóthy, and A. Kocsor: 2005, 'The Szeged Treebank'. In: V. Matoušek, P. Mautner, and T. Pavelka (eds.): TSD, Vol. 3658 of Lecture Notes in Computer Science. pp. 123-131, Springer.

de Marneffe, M.-C. and C. D. Manning: 2008, 'Stanford typed dependencies manual'.

Džeroski, S., T. Erjavec, N. Ledinek, P. Pajas, Z. Žabokrtský, and A. Žele: 2006, 'Towards a Slovene Dependency Treebank'. In: Proceedings of the Fifth International Language Resources and Evaluation Conference, LREC 2006. Genova, Italy, pp. 1388-1391, European Language Resources Association (ELRA).

Hajič, J., J. Panevová, E. Hajičová, P. Sgall, P. Pajas, J. Štěpánek, J. Havelka, M. Mikulová, Z. Žabokrtský, and M. Ševčíková-Razímová: 2006, 'Prague Dependency Treebank 2.0'. CD-ROM, Linguistic Data Consortium, LDC Catalog No.: LDC2006T01, Philadelphia.

Hajič, J., M. Ciaramita, R. Johansson, D. Kawahara, M. A. Martí, L. Màrquez, A. Meyers, J. Nivre, S. Padó, J. Štěpánek, P. Straňák, M. Surdeanu, N. Xue, and Y. Zhang: 2009, 'The CoNLL-2009 Shared Task: Syntactic and Semantic Dependencies in Multiple Languages'. In: Proceedings of the 13th Conference on Computational Natural Language Learning (CoNLL-2009), June 4-5. Boulder, Colorado, USA.

Haverinen, K., T. Viljanen, V. Laippala, S. Kohonen, F. Ginter, and T. Salakoski: 2010, 'Treebanking Finnish'. In: M. Dickinson, K. Müürisep, and M. Passarotti (eds.): Proceedings of the Ninth International Workshop on Treebanks and Linguistic Theories (TLT9). pp. 79-90.

Hudson, R.: 2004, 'Are Determiners Heads?'. Functions of Language 11(1).

Hudson, R.: 2010, An Encyclopedia of Word Grammar and English Grammar. London, UK: University College London, http://tinyurl.com/wg-encyc.

Husain, S., P. Mannem, B. Ambati, and P. Gadde: 2010, 'The ICON-2010 tools contest on Indian language dependency parsing'. In: Proceedings of ICON-2010 Tools Contest on Indian Language Dependency Parsing. Kharagpur, India.

Hwa, R., P. Resnik, A. Weinberg, C. I. Cabezas, and O. Kolak: 2005, 'Bootstrapping parsers via syntactic projection across parallel texts.'. Natural Language Engineering 11(3), 311-325.

Kawata, Y. and J. Bartels: 2000, 'Stylebook for the Japanese Treebank in Verbmobil'. In: Report 240. Tübingen, Germany.

Kromann, M. T., L. Mikkelsen, and S. K. Lynge: 2004, 'Danish Dependency Treebank'. 
Marcus, M. P., B. Santorini, and M. A. Marcinkiewicz: 1993, 'Building a Large Annotated Corpus of English: The Penn Treebank'. Computational Linguistics 19(2), 313-330.

Mareček, D. and Z. Žabokrtský: 2012, 'Exploiting Reducibility in Unsupervised Dependency Parsing'. In: Proceedings of EMNLP-CoNLL'12. pp. 297-307.

McDonald, R., J. Nivre, Y. Quirmbach-Brundage, Y. Goldberg, D. Das, K. Ganchev, K. Hall, S. Petrov, H. Zhang, O. Tc̈kström, C. Bedini, N. B. Castelló, and J. Lee: 2013, 'Universal Dependency Annotation for Multilingual Parsing'. In: Proceedings of the ACL 2013. Association for Computational Linguistics.

McDonald, R., S. Petrov, and K. Hall: 2011a, 'Multi-source Transfer of Delexicalized Dependency Parsers'. In: Proceedings of the Conference on Empirical Methods in Natural Language Processing. Stroudsburg, PA, USA, pp. 62-72, Association for Computational Linguistics.

McDonald, R., S. Petrov, and K. Hall: 2011b, 'Multi-Source Transfer of Delexicalized Dependency Parsers'. In: Proceedings of the 2011 Conference on Empirical Methods in Natural Language Processing. Edinburgh, Scotland, UK., pp. 62-72, Association for Computational Linguistics.

Mel'čuk, I. A.: 1988, Dependency Syntax: Theory and Practice. State University of New York Press.

Montemagni, S., F. Barsotti, M. Battista, N. Calzolari, O. Corazzari, A. Lenci, A. Zampolli, F. Fanciulli, M. Massetani, R. Raffaelli, R. Basili, M. T. Pazienza, D. Saracino, F. Zanzotto, N. Mana, F. Pianesi, and R. Delmonte: 2003, 'Building the Italian Syntactic-Semantic Treebank'. In: A. Abeillé (ed.): Building and using Parsed Corpora. Dordrecht, pp. 189-210, Kluwer.

Nilsson, J., J. Hall, and J. Nivre: 2005, 'MAMBA Meets TIGER: Reconstructing a Swedish Treebank from Antiquity'. In: Proceedings of the NODALIDA Special Session on Treebanks.

Nilsson, J., J. Nivre, and J. Hall: 2006, 'Graph transformations in data-driven dependency parsing'. In: Proceedings of the 21st International Conference on Computational Linguistics and the 44th annual meeting of the Association for Computational Linguistics. pp. 257-264.

Nivre, J., J. Hall, S. Kübler, R. McDonald, J. Nilsson, S. Riedel, and D. Yuret: 2007, 'The CoNLL 2007 Shared Task on Dependency Parsing'. In: Proceedings of the CoNLL 2007 Shared Task. Joint Conf. on Empirical Methods in Natural Language Processing and Computational Natural Language Learning (EMNLP-CoNLL).

Popel, M., D. Mareček, J. Štěpánek, D. Zeman, and Z. Žabokrtský: 2013, 'Coordination Structures in Dependency Treebanks'. In: Proceedings of the 51st Annual Meeting of the Association for Computational Linguistics. Sofia, Bulgaria, pp. 517-527, Association for Computational Linguistics.

Popel, M. and Z. Žabokrtský: 2010, 'TectoMT: modular NLP framework'. Advances in Natural Language Processing pp. 293-304.

Prokopidis, P., E. Desipri, M. Koutsombogera, H. Papageorgiou, and S. Piperidis: 2005, 'Theoretical and practical issues in the construction of a Greek dependency treebank'. In: In Proc. of the 4th Workshop on Treebanks and Linguistic Theories (TLT). pp. 149-160.

Quirk, R., S. Greenbaum, G. Leech, and J. Svartvik: 1985, A Comprehensive Grammar of the English Language. London: Longman.

Ramasamy, L. and Z. Žabokrtský: 2012, 'Prague Dependency Style Treebank for Tamil'. In: Proceedings of LREC 2012. İstanbul, Turkey.

Rasooli, M. S., A. Moloodi, M. Kouhestani, and B. Minaei-Bidgoli: 2011, 'A Syntactic Valency Lexicon for Persian Verbs: The First Steps towards Persian 
Dependency Treebank'. In: 5th Language 83 Technology Conference (LTC): Human Language Technologies as a Challenge for Computer Science and Linguistics. Poznań, Poland, pp. 227-231.

Schwartz, R., O. Abend, and A. Rappoport: 2012, 'Learnability-based Syntactic Annotation Design'. In: Proceedings of COLING 2012: Technical Papers. Mumbai, India, pp. 2405-2422.

Seginer, Y.: 2007, 'Learning Syntactic Structure'. Ph.d. thesis, University of Amsterdam.

Simov, K. and P. Osenova: 2005, 'Extending the Annotation of BulTreeBank: Phase 2'. In: The Fourth Workshop on Treebanks and Linguistic Theories (TLT 2005). Barcelona, pp. 173-184.

Smrž, O., V. Bielický, I. Koư̌ilová, J. Kráčmar, J. Hajič, and P. Zemánek: 2008, 'Prague Arabic Dependency Treebank: A Word on the Million Words'. In: Proceedings of the Workshop on Arabic and Local Languages (LREC 2008). Marrakech, Morocco, pp. 16-23, European Language Resources Association.

Surdeanu, M., R. Johansson, A. Meyers, L. Màrquez, and J. Nivre: 2008, 'The CoNLL-2008 Shared Task on Joint Parsing of Syntactic and Semantic Dependencies'. In: Proceedings of CoNLL.

Taulé, M., M. A. Martí, and M. Recasens: 2008, 'AnCora: Multilevel Annotated Corpora for Catalan and Spanish'. In: LREC. European Language Resources Association.

Tesnière, L.: 1959, Éléments de syntaxe structurale. Paris: Klincksieck.

Tsarfaty, R., J. Nivre, and E. Andersson: 2011, 'Evaluating Dependency Parsing: Robust and Heuristics-Free Cross-Annotation Evaluation'. In: Proceedings of the 2011 Conference on Empirical Methods in Natural Language Processing. Edinburgh, Scotland, UK., pp. 385-396, Association for Computational Linguistics.

van der Beek, L., G. Bouma, J. Daciuk, T. Gaustad, R. Malouf, G. van Noord, R. Prins, and B. Villada: 2002, 'Chapter 5. The Alpino Dependency Treebank'. In: Algorithms for Linguistic Processing NWO PIONIER Progress Report. Groningen, The Netherlands.

Zeman, D.: 2008, 'Reusable Tagset Conversion Using Tagset Drivers'. In: N. Calzolari, K. Choukri, B. Maegaard, J. Mariani, J. Odijk, S. Piperidis, and D. Tapias (eds.): Proceedings of the Sixth International Language Resources and Evaluation Conference, LREC 2008. Marrakech, Morocco, pp. 28-30, European Language Resources Association (ELRA).

Zeman, D., D. Mareček, M. Popel, L. Ramasamy, J. Štěpánek, Z. Žabokrtský, and J. Hajič: 2012, 'HamleDT: To Parse or Not to Parse?'. In: N. Calzolari, K. Choukri, T. Declerck, M. U. Doğan, B. Maegaard, J. Mariani, J. Odijk, and S. Piperidis (eds.): Proceedings of the Eight International Conference on Language Resources and Evaluation (LREC'12). İstanbul, Turkey, European Language Resources Association (ELRA). 


\section{Appendix}

\section{A. List of included languages and treebanks}

- Arabic [ar]: Prague Arabic Dependency Treebank 1.0 / CoNLL 2007 (Smrž et al., 2008)

http://padt-online.blogspot.com/2007/01/conll-shared-task-2007.html

- Basque [eu]: Basque Dependency Treebank, a larger version than the one included in CoNLL 2007, generously provided by IXA Group (Aduriz et al., 2003) http://hdl .handle.net/10230/17098

- Bengali [bn], Hindi [hi] and Telugu [te]: Hyderabad Dependency Treebank / ICON 2010 (Husain et al., 2010)

http://ltrc.iiit.ac.in/icon/2010/nlptools/

- Bulgarian [bg]: BulTreeBank (Simov and Osenova, 2005)

http://www. bultreebank. org/indexBTB.html

- Catalan [ca] and Spanish [es]: AnCora (Taulé et al., 2008)

http://clic.ub.edu/corpus/en/ancora-descarregues

- Czech [cs]: Prague Dependency Treebank 2.0 / CoNLL 2009 (Hajič et al., 2006)

http://ufal.mff.cuni.cz/pdt2.0/

- Danish [da]: Danish Dependency Treebank / CoNLL 2006 (Kromann et al., 2004), now part of the Copenhagen Dependency Treebank

http://code.google.com/p/copenhagen-dependency-treebank/

- Dutch [nl]: Alpino Treebank / CoNLL 2006 (van der Beek et al., 2002)

http://odur.let.rug.nl/ vannoord/trees/

- English [en]: Penn TreeBank 3 / CoNLL 2007 (Marcus et al., 1993) http://www.cis.upenn.edu/ treebank/

- Estonian [et]: Eesti keele puudepank / Arborest (Bick et al., 2004) http://www.cs.ut.ee/ kaili/Korpus/puud/

- Finnish [fi]: Turku Dependency Treebank (Haverinen et al., 2010) http://bionlp.utu.fi/fintreebank.html

- German [de]: Tiger Treebank / CoNLL 2009 (Brants et al., 2004) http://www.ims . uni-stuttgart.de/forschung/ressourcen/korpora/tiger.html

- Greek (modern) [el]: Greek Dependency Treebank (Prokopidis et al., 2005)

http://gdt.ilsp.gr/ 
- Greek (ancient) [grc] and Latin [la]: Ancient Greek and Latin Dependency Treebanks (Bamman and Crane, 2011)

http://nlp.perseus.tufts.edu/syntax/treebank/greek.html, http://nlp.perseus.tufts.edu/syntax/treebank/latin.html

- Hindi [hi]: see Bengali

- Hungarian [hu]: Szeged Treebank (Csendes et al., 2005) http://www.inf.u-szeged.hu/projectdirs/hlt/index_en.html

- Italian [it]: Italian Syntactic-Semantic Treebank / CoNLL 2007 (Montemagni et al., 2003) http://medialab.di.unipi.it/isst/

- Japanese [ja]: Verbmobil (Kawata and Bartels, 2000) http://www.sfs . uni-tuebingen.de/en/tuebajs.shtml

- Latin [la]: see Greek (ancient)

- Persian [fa]: Persian Dependency Treebank (Rasooli et al., 2011) http://dadegan.ir/en/persiandependencytreebank

- Portuguese [pt]: Floresta sintá(c)tica (Afonso et al., 2002) http://www.linguateca.pt/floresta/info_floresta_English.html

- Romanian [ro]: Romanian Dependency Treebank (Călăcean, 2008) http://www.phobos.ro/roric/texts/xml/

- Russian [ru]: Syntagrus (Boguslavsky et al., 2000) http://ruscorpora.ru/en/

- Slovene [sl]: Slovene Dependency Treebank / CoNLL 2006 (Džeroski et al., 2006) http://nl.ijs.si/sdt/

- Spanish [es]: see Catalan

- Swedish [sv]: Talbanken05 (Nilsson et al., 2005) http://www.msi.vxu.se/users/nivre/research/Talbanken05.html

- Tamil [ta]: TamilTB (Ramasamy and Žabokrtský, 2012) http://ufal.mff.cuni.cz/ ramasamy/tamiltb/0.1/

- Telugu [te]: see Bengali

- Turkish [tr]: METU-Sabanci Turkish Treebank (Atalay et al., 2003) http://ii.metu.edu.tr/corpus/ 


\section{B. Examples of harmonization of dependency relations}

\begin{tabular}{|c|c|c|}
\hline orig. label & tokens & distribution of HamleDT v1.5 labels \\
\hline $\mathrm{k} 1$ & 1,168 & $\mathrm{Sb}=98 \%$ Coord $=2 \%$ \\
\hline main & 1,130 & Pred $=85 \%$ Coord $=14 \%$ \\
\hline r6 & 790 & Atr $=98 \%$ Coord $=2 \%$ \\
\hline $\mathrm{k} 2$ & 788 & Obj $=95 \%$ Coord $=5 \%$ \\
\hline ccof & 602 & $\begin{array}{l}\text { Pred }=40 \% \quad \text { Atr }=23 \% \quad \text { Obj=12\% } \\
\text { Adv }=8 \% \text { Cb }=7 \% \text { Pnom }=2 \%\end{array}$ \\
\hline vmod & 583 & $\mathrm{Adv}=98 \%$ Coord $=2 \%$ \\
\hline pof & 421 & $\mathrm{Obj}=100 \%$ \\
\hline $\mathrm{k} 7 \mathrm{p}$ & 325 & $\mathrm{Adv}=98 \%$ Coord $=2 \%$ \\
\hline $\mathrm{k} 7 \mathrm{t}$ & 303 & $\mathrm{Adv}=100 \%$ \\
\hline nmod & 233 & Atr $=91 \%$ Coord $=9 \%$ \\
\hline k1s & 202 & Pnom $=98 \%$ Coord $=2 \%$ \\
\hline k7 & 152 & $\mathrm{Adv}=97 \%$ Coord $=3 \%$ \\
\hline other & 470 & 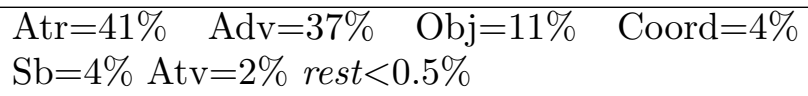 \\
\hline
\end{tabular}

Table IV.: The Bengali treebank [bn] uses 42 dependency labels, but we show only 12 most frequent ones. The remaining 30 labels are summarized in the last line. Bengali dependency labels are explained in http://ltrc.iiit.ac.in/nlptools2010/files/documents/dep-tagset.pdf and their mapping to HamleDT v1.5 dependency labels is relatively straightforward, except for coordinations, where the Bengali treebank marks the conjunction with the dependency relation, while in HamleDT v1.5, the conjuncts are marked with the dependency relation and the conjunction is marked with Coord). For example, $\mathrm{k} 7 \mathrm{p}$ is location in space, $\mathrm{k} 7 \mathrm{t}$ location in time and $\mathrm{k} 7$ location elsewhere; all three labels are basically mapped to $A d v$ (adverbial). 


\begin{tabular}{|c|c|c|}
\hline orig. label & tokens & distribution of HamleDT v1.5 labels \\
\hline NMOD & 155,951 & $\begin{array}{l}\mathrm{Atr}=62 \% \text { AuxA=22\% AuxP=13\% Coord }=1 \% \text { AuxV=1\% } \\
\text { rest }=1 \%\end{array}$ \\
\hline $\mathrm{P}$ & 52,051 & AuxX $=44 \%$ AuxK=36\% AuxG=20\% rest $<0.5 \%$ \\
\hline PMOD & 45,207 & $\begin{array}{lllll}\mathrm{Adv}=50 \% & \text { Atr }=40 \% & \text { Coord }=6 \% & \mathrm{AuxP}=2 \% & \mathrm{NR}=1 \% \\
\mathrm{Obj}=1 \% \text { rest }<0.5 \% & & & \\
\end{array}$ \\
\hline SBJ & 35,446 & $\begin{array}{l}\mathrm{Sb}=94 \% \text { Coord }=2 \% \quad \mathrm{NR}=1 \% \text { Atr }=1 \% \text { Obj=1\% } \mathrm{Adv}=1 \% \\
\text { rest }<0.5 \%\end{array}$ \\
\hline ADV & 32,202 & 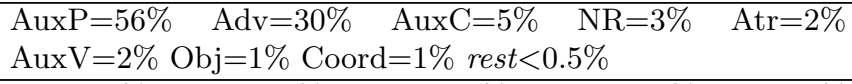 \\
\hline OBJ & 30,507 & $\begin{array}{lllll}\text { Obj }=55 \% & \mathrm{Adv}=29 \% & \mathrm{AuxV}=8 \% & \text { Coord }=5 \% & \mathrm{Atr}=2 \% \\
\text { rest }=1 \% & & & & \\
\end{array}$ \\
\hline COORD & 22,865 & $\begin{array}{llllr}\operatorname{Atr}=34 \% & \mathrm{Adv}=21 \% & \mathrm{Obj}=12 \% & \text { Pred }=11 \% & \mathrm{Sb}=8 \% \\
\mathrm{AuxV}=5 \% & \text { Pnom=3\% } & \text { AuxP=2\% } & \mathrm{NR}=2 \% & \text { Coord }=1 \% \\
\text { AuxA }=1 \% & \text { rest=1\% } & & & \\
\end{array}$ \\
\hline VMOD & 21,053 & $\begin{array}{l}\text { AuxV=30\% AuxC=24\% Pnom=20\% Neg=10\% } \quad \text { Adv }=6 \% \\
\text { Atr }=3 \% \text { Coord }=2 \% \text { Obj=2\% NR=2\% AuxP=1\% Sb=1\% } \\
\text { rest }<0.5 \%\end{array}$ \\
\hline ROOT & 18,791 & Pred $=69 \%$ AuxV $=18 \%$ Coord $=8 \%$ ExD $=4 \%$ rest $<0.5 \%$ \\
\hline AMOD & 15,269 & $\begin{array}{lllll}\operatorname{Atr}=52 \% & \text { Adv }=19 \% & \text { AuxP }=14 \% & \text { NR=9\% } & \text { AuxC }=3 \% \\
\text { AuxV }=1 \% & \text { rest }=1 \% & & & \\
\end{array}$ \\
\hline $\mathrm{VC}$ & 13,745 & $\begin{array}{l}\text { Pred }=29 \% \text { Adv }=25 \% \text { Obj=23\% AuxV=10\% Atr }=9 \% \text { Co- } \\
\text { ord }=2 \% \text { NR }=1 \% \text { rest }<0.5 \%\end{array}$ \\
\hline IOBJ & 1,883 & $\mathrm{Obj}=92 \% \mathrm{Adv}=3 \%$ Coord $=2 \%$ Atr $=1 \% \mathrm{Sb}=1 \%$ rest $<0.5 \%$ \\
\hline $\mathrm{CC}$ & 1,336 & $\mathrm{NR}=98 \% \mathrm{Neg}=2 \%$ \\
\hline PRT & 1,268 & $\mathrm{AuxV}=95 \%$ AuxC $=2 \% \mathrm{Adv}=2 \%$ rest $<0.5 \%$ \\
\hline PRN & 1,259 & $\begin{array}{l}\text { Atr }=43 \% \quad \text { Adv }=27 \% \quad \text { AuxP }=8 \% \quad \mathrm{NR}=7 \% \\
\text { ord }=5 \% \text { OuxV }=1 \% \text { AuxC }=1 \% \text { rest }<0.5 \%\end{array}$ \\
\hline LGS & 1,211 & $\mathrm{AuxP}=99 \%$ AuxC $=1 \%$ rest $<0.5 \%$ \\
\hline DEP & 892 & $\begin{array}{lllll}\mathrm{AuxP}=46 \% & \mathrm{Atr}=23 \% & \mathrm{Adv}=10 \% & \mathrm{NR}=9 \% & \mathrm{Neg}=4 \% \\
\mathrm{AuxC}=3 \% \text { AuxA }=2 \% \text { Coord }=1 \% \text { rest }=1 \% & \\
\end{array}$ \\
\hline GAP & 272 & $\begin{array}{lllll}\operatorname{Atr}=47 \% & \text { AuxP }=38 \% & \mathrm{Adv}=10 \% & \mathrm{NR}=2 \% & \text { Coord }=1 \% \\
\text { AuxC }=1 \% & \mathrm{Neg}=1 \%\end{array}$ \\
\hline EXP & 219 & $\mathrm{Adv}=84 \%$ AuxV=11\% Coord $=5 \%$ \\
\hline TMP & 149 & $\operatorname{Atr}=97 \% \mathrm{NR}=3 \%$ \\
\hline
\end{tabular}

Table V.: The English treebank [en] (from CoNLL 2007) uses 20 dependency labels, but their mapping to HamleDT v1.5 labels is not straightforward. In practice, we found the English CoNLL 2007 labels not helpful, and we based the conversion only on dependency structure and morphological tags. 


\section{List of dependency relation labels in figures}

\begin{tabular}{|c|c|c|c|}
\hline Language & Label & Description & Example \\
\hline & $\mathbf{X}$ & $\begin{array}{l}\text { Our meta-label that represents the unknown } \\
\text { relation of the depicted subtree to its unshown } \\
\text { parent. }\end{array}$ & \\
\hline $\mathrm{bg}$ & comp & $\begin{array}{l}\text { Complement, i.e. argument of non-verbal } \\
\text { head, non-finite verbal head, copula. }\end{array}$ & Figure 18 \\
\hline bg & indobj & Child is indirect object of parent. & Figure 18 \\
\hline bg & $\bmod$ & $\begin{array}{l}\text { Child is modifier, e.g. of a noun phrase, or a } \\
\text { negative particle modifying a verb etc. }\end{array}$ & Figure 18 \\
\hline $\mathrm{bg}$ & prepcomp & Child is noun phrase, parent is preposition. & Figure 18 \\
\hline $\mathrm{bg}$ & subj & Child is subject of parent. & Figure 18 \\
\hline$\overline{b g}$ & xcomp & $\begin{array}{l}\text { Child is clausal complement; this includes } \\
\text { complements of modal verbs. }\end{array}$ & Figure 18 \\
\hline $\mathrm{ca}$ & $\mathrm{CO}$ & $\begin{array}{l}\text { Child is coordinating conjunction, parent is } \\
\text { the first conjunct. }\end{array}$ & Figure 4 \\
\hline ca & CONJUNCT & $\begin{array}{l}\text { Parent is the first conjunct, child is one of the } \\
\text { other conjuncts. }\end{array}$ & Figure 4 \\
\hline ca & PUNC & Child is punctuation symbol. & Figure 4 \\
\hline cs, sl, la, ta & $\mathrm{Adv}$ & Child is adverbial modifier of parent. & Figure 2 \\
\hline cs, sl, la, ta & Atr & Parent is noun, child is its attribute. & Figure 9 \\
\hline cs, sl, la, ta & AuxC & $\begin{array}{l}\text { Child is subordinating conjunction, parent is } \\
\text { governing predicate. The relation of the sub- } \\
\text { ordinate clause to the parent is labeled at the } \\
\text { grandchild. }\end{array}$ & Figure 19 \\
\hline cs, sl, la, ta & AuxP & $\begin{array}{l}\text { Child is preposition. The relation of the prepo- } \\
\text { sitional phrase to the parent is labeled at the } \\
\text { grandchild. }\end{array}$ & Figure 2 \\
\hline cs, sl, la, ta & AuxV & $\begin{array}{l}\text { Child is auxiliary verb or negative particle, } \\
\text { parent is content verb. }\end{array}$ & Figure 19 \\
\hline cs, sl, la, ta & AuxX & $\begin{array}{l}\text { Child is comma and does not serve as coordi- } \\
\text { nation root. }\end{array}$ & Figure 2 \\
\hline cs, sl, la, ta & AuxZ & Emphasizing word. & Figure 8 \\
\hline cs, sl, la, ta & Coord & Child serves as root of a coordinate structure. & Figure 1 \\
\hline cs, sl, la, ta & Obj & Child is object of parent. & Figure 2 \\
\hline cs, sl, la, ta & Pred & Child is predicate of a main clause. & Figure 2 \\
\hline cs, sl, la, ta & $\mathrm{Sb}$ & Child is subject of parent. & Figure 19 \\
\hline $\mathrm{cs}, \mathrm{ta}$ & - M & $\begin{array}{l}\text { Suffix to a label, saying that the child is a } \\
\text { conjunct. The main label tags its relation to } \\
\text { the parent of the coordinate structure. }\end{array}$ & Figure 1 \\
\hline da & appr & Restrictive apposition (no comma). & Figure 28 \\
\hline da & conj & $\begin{array}{l}\text { Child is conjunct, parent is first conjunct or } \\
\text { coordinating conjunction. }\end{array}$ & Figure 6 \\
\hline da & coord & $\begin{array}{l}\text { Parent is conjunct, child is coordinating con- } \\
\text { junction. }\end{array}$ & Figure 6 \\
\hline $\mathrm{da}$ & dobj & Child is direct object of parent. & Figure 28 \\
\hline
\end{tabular}




\begin{tabular}{|c|c|c|c|}
\hline da & expl & Child is expletive subject of parent. & Figure 28 \\
\hline da & $\bmod$ & $\begin{array}{l}\text { Modifier, e.g. attribute of noun, adverbial } \\
\text { modifier of verb, adjective attached to deter- } \\
\text { miner etc. }\end{array}$ & Figure 28 \\
\hline da & nobj & $\begin{array}{l}\text { Child is noun phrase or infinitive, parent is e.g. } \\
\text { determiner, numeral, preposition etc. }\end{array}$ & Figure 28 \\
\hline da & pnct & Child is punctuation symbol. & Figure 6 \\
\hline $\mathrm{da}$ & possd & $\begin{array}{l}\text { Child is argument of possessive parent, i.e. } \\
\text { child is the thing possessed. }\end{array}$ & Figure 28 \\
\hline de & CD & $\begin{array}{l}\text { Child is coordinating conjunction, parent is } \\
\text { one conjunct and right sibling is the other } \\
\text { conjunct. }\end{array}$ & Figure 3 \\
\hline de & CJ & Parent and child are conjuncts. & Figure 3 \\
\hline de & $\mathrm{MO}$ & $\begin{array}{l}\text { Modifier. In NPs only focus particles are an- } \\
\text { notated as modifiers. }\end{array}$ & Figure 23 \\
\hline de & NG & $\begin{array}{l}\text { Child is negative particle, parent is negated } \\
\text { verb. }\end{array}$ & Figure 23 \\
\hline de & NK & $\begin{array}{l}\text { Noun Kernel. Child attached within a noun } \\
\text { phrase or a prepositional phrase. }\end{array}$ & Figure 10 \\
\hline de & OA & Child is accusative object of parent. & Figure 23 \\
\hline de & $\mathrm{OC}$ & $\begin{array}{l}\text { Clausal object. Also verb tokens building a } \\
\text { complex verbal form and modal constructions. }\end{array}$ & Figure 23 \\
\hline de & PUNC & Child is punctuation symbol. & Figure 3 \\
\hline $\mathrm{de}$ & SB & Child is subject of parent. & Figure 23 \\
\hline es & atr & $\begin{array}{l}\text { Attribute. E.g. child is adverbial/prepositional } \\
\text { phrase, parent is verb. }\end{array}$ & Figure 12 \\
\hline es & $\mathrm{cd}$ & Child is direct object of parent. & Figure 12 \\
\hline es & conj & Child is subordinating conjunction. & Figure 12 \\
\hline es & s.a & Child is adjectival phrase, parent is not verb. & Figure 12 \\
\hline es & sn & $\begin{array}{l}\text { Child is noun phrase. Parent may be e.g. } \\
\text { preposition. }\end{array}$ & Figure 12 \\
\hline es & spec & $\begin{array}{l}\text { Specifier. E.g. child is determiner and parent } \\
\text { is noun. }\end{array}$ & Figure 12 \\
\hline es & suj & Child is subject of parent. & Figure 12 \\
\hline fa & NPREMOD & Child is premodifier of parent noun. & Figure 26 \\
\hline fa & NVE & $\begin{array}{l}\text { Child is non-verbal element of compound verb. } \\
\text { Parent is verbal element. }\end{array}$ & Figure 26 \\
\hline $\mathrm{fa}$ & SBJ & Child is subject of parent. & Figure 26 \\
\hline hi & lwg_cont & $\begin{array}{l}\text { Child is additional node of a complex expres- } \\
\text { sion; child and parent together perform certain } \\
\text { function. }\end{array}$ & Figure 27 \\
\hline hi & lwg_psp & Child is postposition and modifies a noun. & Figure 11 \\
\hline hi & lwg_vaux & Child is auxiliary verb, parent is content verb. & Figure 27 \\
\hline hi & pof & Part of relation, e.g. part of conjunct verb. & Figure 27 \\
\hline hi & pof_cn & Part of relation. & Figure 27 \\
\hline hi, bn, te & $\mathrm{adv}$ & $\begin{array}{l}\text { Child is adverbial modifier (only adverbs of } \\
\text { manner) of parent. }\end{array}$ & Figure 29 \\
\hline
\end{tabular}




\begin{tabular}{|c|c|c|c|}
\hline hi, bn, te & ccof & $\begin{array}{l}\text { Child is conjunct, parent is coordinating con- } \\
\text { junction or comma. }\end{array}$ & Figure 29 \\
\hline hi, bn, te & $\mathrm{k} 1$ & $\begin{array}{l}\text { Child is karta (doer / agent / subject) of } \\
\text { parent predicate. }\end{array}$ & Figure 27 \\
\hline hi, bn, te & $\mathrm{k} 2$ & $\begin{array}{l}\text { Child is karma (pacient / object) of parent } \\
\text { predicate. }\end{array}$ & Figure 27 \\
\hline hi, bn, te & $\mathrm{k} 7 \mathrm{p}$ & $\begin{array}{l}\text { Child is deshadhikarana (location in space) of } \\
\text { the parent predicate. }\end{array}$ & Figure 30 \\
\hline hi, bn, te & $\mathrm{k} 7 \mathrm{t}$ & $\begin{array}{l}\text { Child is kaalaadhikarana (location in time) of } \\
\text { the parent predicate. }\end{array}$ & Figure 31 \\
\hline hi, bn, te & nmod & Parent is noun, child is its attribute. & Figure 29 \\
\hline hi, bn, te & nmod_adj & Child is adjective and modifies a noun. & Figure 11 \\
\hline hi, bn, te & r6 & $\begin{array}{l}\text { Shashthi (possessive). Child is possessor in } \\
\text { genitive, parent is the possessed noun. }\end{array}$ & Figure 30 \\
\hline hu & ATT & Attribute. & Figure 15 \\
\hline $\mathrm{hu}$ & CONJ & $\begin{array}{l}\text { Child is conjunction (coordinating or subordi- } \\
\text { nating). }\end{array}$ & Figure 5 \\
\hline hu & DET & Child is determiner, parent is noun. & Figure 15 \\
\hline $\mathrm{hu}$ & ILL & Child is verbal argument in illative case. & Figure 15 \\
\hline hu & OBJ & Child is object of parent. & Figure 15 \\
\hline hu & PUNCT & Child is punctuation symbol. & Figure 5 \\
\hline hu & SUBJ & Child is subject of parent. & Figure 15 \\
\hline it & cong_sub & Parent is subordinating conjunction. & Figure 13 \\
\hline it & det & Child is determiner, parent is noun. & Figure 13 \\
\hline it & modal & $\begin{array}{l}\text { Child is modal (dovere, volere, potere) or as- } \\
\text { pectual (andare, venire, stare) verb, parent is } \\
\text { content verb. }\end{array}$ & Figure 13 \\
\hline it & pred & $\begin{array}{l}\text { Parent is verb (often it is copula), child is } \\
\text { predicative complement (nominal predicate). }\end{array}$ & Figure 13 \\
\hline it & $\operatorname{sogg}$ & Child is subject of parent. & Figure 13 \\
\hline$\overline{\mathrm{j} \text { ja }}$ & $\begin{array}{l}\text { ADJ } \\
\end{array}$ & Child is adjunct of parent. & Figure 25 \\
\hline ja & COMP & $\begin{array}{l}\text { Complement, e.g. verb attached to another } \\
\text { verb form, noun attached to postposition etc. }\end{array}$ & Figure 25 \\
\hline ja & SBJ & Child is subject of parent. & Figure 25 \\
\hline $\mathrm{nl}$ & det & Child is determiner, parent is noun. & Figure 21 \\
\hline $\mathrm{nl}$ & $\bmod$ & $\begin{array}{l}\text { Child is adverbial modifier (bijwoordelijke } \\
\text { bepaling) of parent. }\end{array}$ & Figure 21 \\
\hline $\mathrm{nl}$ & obj1 & $\begin{array}{l}\text { Child is direct object; this includes nouns } \\
\text { attached to prepositions! }\end{array}$ & Figure 21 \\
\hline $\mathrm{nl}$ & predm & $\begin{array}{l}\text { Child determines state (adverbial modifier), } \\
\text { parent is predicate. }\end{array}$ & Figure 22 \\
\hline $\mathrm{nl}$ & $\mathrm{su}$ & Child is subject of parent. & Figure 21 \\
\hline $\mathrm{nl}$ & vc & $\begin{array}{l}\text { Verbal complement. Example: parent is } \\
\text { modal, child is infinitive. }\end{array}$ & Figure 21 \\
\hline $\mathrm{pt}$ & $>\mathrm{N}$ & Child is left dependent of nominal core. & Figure 24 \\
\hline $\mathrm{pt}$ & ADVL & $\begin{array}{l}\text { Child is adverbial adjunct (adjunto adverbial) } \\
\text { of parent. }\end{array}$ & Figure 24 \\
\hline
\end{tabular}




\begin{tabular}{|l|l|l|l|}
\hline $\mathrm{pt}$ & MV & $\begin{array}{l}\text { Child is main verb, parent may be e.g. modal } \\
\text { verb. }\end{array}$ & Figure 24 \\
\hline $\mathrm{pt}$ & $\mathrm{N}<$ & Child is right dependent of nominal core. & Figure 24 \\
\hline $\mathrm{pt}$ & $\mathrm{P}<$ & Child is right dependent of preposition. & Figure 24 \\
\hline $\mathrm{pt}$ & PRT-AUX $<$ & $\begin{array}{l}\text { Child is verbal particle (partícula de ligação } \\
\text { verbal), e.g. between modal and content verb, } \\
\text { parent would be modal. }\end{array}$ & Figure 24 \\
\hline $\mathrm{pt}$ & PUNC & Child is punctuation symbol. & Figure 24 \\
\hline $\mathrm{pt}$ & SC & $\begin{array}{l}\text { Child is nominal predicate (predicativo do } \\
\text { sujeito), parent is copula. }\end{array}$ & Figure 24 \\
\hline $\mathrm{pt}$ & SUBJ & Child is subject of parent. & Figure 24 \\
\hline $\mathrm{ro}$ & rel.conj. & $\begin{array}{l}\text { Parent is coordinating conjunction, child is } \\
\text { conjunct. }\end{array}$ & Figure 7 \\
\hline \hline $\mathrm{ru}$ & 1-компл & $\begin{array}{l}\text { Child is argument other than subject. Also: } \\
\text { genitive noun modifier of another noun. }\end{array}$ & Figure 17 \\
\hline $\mathrm{ru}$ & агент & Child is agent-object of passive parent. & Figure 17 \\
\hline $\mathrm{ru}$ & опред & Parent is noun, child is its attribute. & Figure 17 \\
\hline $\mathrm{ru}$ & пасс-анал & $\begin{array}{l}\text { Child is passive participle, parent is finite } \\
\text { auxiliary verb. }\end{array}$ & Figure 17 \\
\hline $\mathrm{ru}$ & предик & Parent is predicate, child is subject. & Figure 17 \\
\hline \hline $\mathrm{ta}$ & АСотр & $\begin{array}{l}\text { Child is (obligatory) adverbial complement of } \\
\text { parent. }\end{array}$ & Figure 8 \\
\hline \hline $\mathrm{tr}$ & ОВJECT & Child is object of parent. & Figure 16 \\
\hline $\mathrm{tr}$ & $\begin{array}{l}\text { QUESTION } \\
\text {.РАRTICLE }\end{array}$ & $\begin{array}{l}\text { Child is question particle, parent is verb. } \\
\text { SUВJECT }\end{array}$ & Child is subject of parent. \\
\hline $\mathrm{tr}$ & VOCATIVE & $\begin{array}{l}\text { Child is vocative noun phrase serving as doer } \\
\text { (actor) of parent verb. }\end{array}$ & Figure 16 \\
\hline
\end{tabular}


paper.tex; 2/07/2014; 18:00; p.40 